\title{
On initial-boundary value problem for nonlinear integro-differential equations with variable exponents of nonlinearity
}

\author{
Oleh Buhrii ${ }^{1}$, Nataliya Buhrii ${ }^{2}$ \\ ${ }^{1}$ Ivan Franko National University of Lviv, Lviv, Ukraine \\ ${ }^{2}$ Lviv Polytechnic National University, Lviv, Ukraine
}

Received: 5 December 2016, Accepted: 27 February 2017

Published online: 25 August 2017.

\begin{abstract}
Some nonlinear parabolic integro-differential equations with variable exponents of the nonlinearity are considered. The initial-boundary value problem for these equations is investigated and the existence theorem for the problem is proved.
\end{abstract}

Keywords: Nonlinear parabolic equation, integro-differential equation, generalized Lebesgue space, variable exponents of nonlinearity.

\section{Introduction}

Let $n \in \mathbb{N}$ and $T>0$ be fixed numbers, $\Omega \subset \mathbb{R}^{n}$ be a bounded domain with the boundary $\partial \Omega, Q_{0, T}:=\Omega \times(0, T)$, $\Sigma_{0, T}:=\partial \Omega \times(0, T), \Omega_{\tau}:=\{(x, t) \mid x \in \Omega, t=\tau\}, \tau \in \mathbb{R}$. We seek a weak solution $u: Q_{0, T} \rightarrow \mathbb{R}^{1}$ of the problem

$$
\begin{gathered}
|u|^{r-2} u_{t}-a \Delta u+g(x, t)|u|^{q(x)-2} u+\phi(E u)=f(x, t), \quad(x, t) \in Q_{0, T}, \\
\left.u\right|_{\Sigma_{0, T}}=0,\left.\quad u\right|_{t=0}=u_{0}(x), \quad x \in \Omega,
\end{gathered}
$$

where $a>0$ and $r>1$ are some numbers, $\Delta:=\frac{\partial^{2}}{\partial x_{1}^{2}}+\frac{\partial^{2}}{\partial x_{2}^{2}}+\ldots+\frac{\partial^{2}}{\partial x_{n}^{2}}$ is the Laplacian,

$$
(E u)(x, t):=\int_{\Omega} \mathfrak{Z}(x, t, z)(\widetilde{u}(x+z, t)-\widetilde{u}(x, t)) d z, \quad(x, t) \in Q_{0, T},
$$

$g, q, \phi, \mathfrak{Z}, f, u_{0}$ are some functions, and $\tilde{u}$ is zero extension of $u$ from $Q_{0, T}$ into $\left(\mathbb{R}^{n} \backslash \Omega\right) \times(0, T)$.

The equations of type (1) have been widely used in many applications. For example (see [4]), let us consider a circuit, which consists of a resistance, a condenser, and a generator of the jump impulses. It is well known that the supply $U(t)$ satisfies the stochastic differential equation

$$
d U(t)=-\frac{1}{R C} U(t) d t+\sigma d W(t)+d Q(t), \quad t \in[0, T], \quad U(0)=U_{0}
$$

where $R$ is a value of the resistance, $C$ is a capacitance of the condenser, $\sigma$ is a intensity of the loss of the signal, $U_{0}$ is a start supply, $T=R C,\{W(t)\}_{t \in[0, T]}$ is the standard Brownian motion, and $Q(t)$ is the compound Poisson process. Let 
$u(x, t), x \in \mathbb{R}$, be a density of the stochastic process $U(t), t \in[0, T]$. If some additional conditions are satisfied, then (see $[4$, p. 4]) $u$ is a solution to the Cauchy problem for the Kolmogorov-Feller equation

$$
u_{t}-\frac{\sigma^{2}}{2} u_{x x}-\frac{1}{R C}(x u)_{x}-\lambda \int_{\mathbb{R}} \psi(\xi, t)(u(x+\xi, t)-u(x, t)) d \xi=0, \quad(x, t) \in \mathbb{R} \times(0, T), \quad u(x, 0)=u_{0}(x), \quad x \in \mathbb{R}
$$

where $u_{0}$ is a density of $U_{0}, \lambda$ is a intensity of the jump creation, $\psi$ is a density of the jump size.

If $n=1$, then equation (1) is a nonlinear modification of (5) in bounded domain $Q_{0, T}$ instead of the unbounded strip $\mathbb{R} \times(0, T)$. Equation (5) also arises in the Merton and Kou models of the option pricing (see [15], [31], and [34]). The Cauchy problem for the equations of type (5) is considered in [9], [16], [21], and [41].

The number $r$ and the function $q$ (see (1)) are called exponents of the nonlinearity of double nonlinear parabolic equation (1). Since $q$ is a function, here we have a variable exponent of the nonlinearity. The double nonlinear parabolic equations with variable exponents of the nonlinearity without the integral terms are considered in [2], [7]. In [6], [17], [18], [19], [39], and [40], the authors investigate the problems for nonlinear parabolic equations with constant exponents of the nonlinearity and with the integral terms which differ from term (3).

In [38], J.P. Pinasco proved the existence of the local solution and the nonexistence of the global solution to the initial-boundary value problem for the equation

$$
u_{t}-\Delta u-|u|^{\alpha(x)}=\int_{\Omega}|u(z, t)|^{\beta(z)} d z
$$

where $\alpha(x)>1$ and $\beta(y)>1$.

In this paper we extend our investigations which is started in [13] for the double nonlinear equation

$$
u_{t}-a \Delta\left(|u|^{\gamma-2} u\right)+g(x, t)|u|^{q(x)-2} u+\phi(E u)=f(x, t), \quad(x, t) \in Q_{0, T}
$$

We recall that if $\gamma \in[2,3)$, then the existence of the weak solution to problem (7), (2) is proved in [13]. The Cauchy problem for the equations of type (7) is considered in [20] if $q(x) \equiv 2, \phi(s)=s$, and $\gamma=3$.

\section{Notation and statement of main result}

Let $(\cdot, \cdot)_{H}$ be a scalar product of some Hilbert space $H,\|\cdot\|_{B} \equiv\|\cdot ; B\|$ a norm of some Banach space $B, B^{*}$ a dual space, and $\langle\cdot, \cdot\rangle_{B}$ a scalar product between $B^{*}$ and $B$. For the Banach spaces $X$ and $Y$ the notation $X \circlearrowleft Y$ means the continuous embedding; the notation $X \bar{\circlearrowleft} Y$ means the continuous and densely embedding; the notation $X \stackrel{K}{\subset} Y$ means the compact embedding.

Suppose that $m, N \in \mathbb{N}, p \in[1, \infty], \mathrm{Q}=\Omega$ or $\mathrm{Q}=Q_{0, T}, \mathscr{M}(\mathrm{Q})$ is a set of all measurable functions $v: \mathrm{Q} \rightarrow \mathbb{R}$ (see $[24$, p. 120]), $\operatorname{Lip}(\mathrm{Q})$ is a set of all Lipschitz-continuous functions $v: Q \rightarrow \mathbb{R}$ (see [32, p. 29]), $C^{m}(\mathrm{Q})$ and $C_{0}^{\infty}(\mathrm{Q})$ are determined from [1, p. 9], $L^{p}(\mathrm{Q})$ is the Lebesgue space (see [1, p. 22, 24]), $W^{m, p}(\mathrm{Q})$ and $W_{0}^{m, p}(\mathrm{Q})$ are Sobolev spaces (see [1, p. 45]), $H^{m}(\mathrm{Q}):=W^{m, 2}(\mathrm{Q}), H_{0}^{m}(\mathrm{Q}):=W_{0}^{m, 2}(\mathrm{Q}), C([0, T] ; X)$ and $C^{m}([0, T] ; X)$ are determined from $[27$, p. 147], $L^{p}(0, T ; X)$ is determined from [27, p. 155], $W^{m, p}(0, T ; X)$ is determined from [25, p. 286], 
$H^{m}(0, T ; X):=W^{m, 2}(0, T ; X)$, and

$$
\mathscr{B}_{+}(\mathrm{Q}):=\left\{\delta \in L^{\infty}(\mathrm{Q}) \mid \underset{y \in Q}{\operatorname{ess} \inf } \boldsymbol{\delta}(y)>0\right\}
$$

For every $\delta \in \mathscr{B}_{+}(\mathrm{Q})$, by definition, put

$$
\begin{gathered}
\delta_{0}:=\underset{y \in Q}{\operatorname{essinf}} \delta(y), \quad \delta^{0}:=\underset{y \in Q}{\operatorname{ess} \sup } \delta(y), \quad S_{\delta}(s):=\max \left\{s^{\delta_{0}}, s^{\delta^{0}}\right\}, \quad s \geq 0, \\
\delta^{\prime}(y):=\frac{\delta(y)}{\delta(y)-1} \text { for a.e. } y \in \mathrm{Q}\left(\text { note that } \frac{1}{\delta(y)}+\frac{1}{\delta^{\prime}(y)}=1 \text { and } \delta^{\prime} \in \mathscr{B}_{+}(\mathrm{Q})\right), \\
\rho_{\delta}(v ; \mathrm{Q}):=\int_{\mathrm{Q}}|v(y)|^{\delta(y)} d y, \quad v \in \mathscr{M}(\mathrm{Q}) .
\end{gathered}
$$

For every function $u \in L^{1}\left(Q_{0, T}\right)=L^{1}\left(0, T ; L^{1}(\Omega)\right)$ we have $u(\cdot, t) \in L^{1}(\Omega), t \in(0, T)$. For the sake of convenience we shall write $u(t)$ instead of $u(\cdot, t)$.

Assume that $\delta \in \mathscr{B}_{+}(\mathrm{Q})$ and $\delta_{0}>1$. The set

$$
L^{\delta(y)}(\mathrm{Q}):=\left\{v \in \mathscr{M}(\mathrm{Q}) \mid \rho_{\delta}(v ; \mathrm{Q})<+\infty\right\}
$$

with the Luxemburg norm

$$
\left\|v ; L^{\delta(y)}(\mathrm{Q})\right\|:=\inf \left\{\lambda>0 \mid \rho_{\delta}(v / \lambda ; \mathrm{Q}) \leq 1\right\}
$$

is called a generalized Lebesgue space. It is well known that $L^{\delta(y)}(\mathrm{Q})$ is the Banach space which is reflexive and separable (see [30, p. 599, 600, 604]). The generalized Lebesgue space was introduced in [36]. Its properties were widely studied in [2], [10], [22], [26], and [30].

We shall need the following assumptions:
(A): $a>0, \frac{3}{2}<r \leq 2$;
(Q): $q \in \mathscr{B}_{+}(\Omega), 1<q_{0} \leq q^{0} \leq 2$;
(G): $g \in \mathscr{B}_{+}\left(Q_{0, T}\right)$;
(E): $\mathfrak{Z} \in L^{\infty}\left(Q_{0, T} \times \Omega\right)$;
$(\Phi): \phi \in \operatorname{Lip}(\mathbb{R}),|\phi(\xi)| \leq \phi^{*}|\xi|$ for every $\xi \in \mathbb{R}$, where $\phi^{*} \in[0,+\infty)$;
(F): $f \in L^{r^{\prime}}\left(Q_{0, T}\right)$, where $\frac{1}{r}+\frac{1}{r^{\prime}}=1$;
(U): $u_{0} \in H^{2}(\Omega) \cap H_{0}^{1}(\Omega)$.

By definition, put

$$
\begin{gathered}
\gamma:=\frac{4}{4-r}\left(\text { note that } \gamma \in\left(\frac{8}{5}, 2\right] \text { iff } r \in\left(\frac{3}{2}, 2\right]\right), \\
(G u)(x, t):=g(x, t)|u(x, t)|^{q(x)-2} u(x, t), \quad(x, t) \in Q_{0, T}, \quad u \in \mathscr{M}\left(Q_{0, T}\right), \\
(\mathscr{R} u)(x, t):=\frac{1}{r-1}|u(x, t)|^{r-2} u(x, t), \quad(x, t) \in Q_{0, T}, \quad u \in \mathscr{M}\left(Q_{0, T}\right) .
\end{gathered}
$$

Notice that if $u \in C^{1}\left(Q_{0, T}\right)$ and $u \neq 0$, then

$$
(\mathscr{R} u)_{t}=|u|^{r-2} u_{t}
$$

Then we formally rewrite equation (1) as

$$
(\mathscr{R} u)_{t}-a \Delta u+G u+\phi(E u)=f(x, t), \quad(x, t) \in Q_{0, T}
$$


Definition 1. A real-valued function $u \in L^{2}\left(0, T ; H_{0}^{1}(\Omega)\right) \cap C\left([0, T] ; L^{\gamma}(\Omega)\right)$ is called a weak solution of problem (1)-(2) if $\mathscr{R} u, G u, E u \in L^{2}\left(Q_{0, T}\right)$, u satisfies (2), and for every $v \in H_{0}^{1}\left(Q_{0, T}\right)$ we have

$$
\int_{Q_{0, T}}\left[-\mathscr{R} u v_{t}+a(\nabla u, \nabla v)+G u v+\phi(E u) v\right] d x d t=\int_{Q_{0, T}} f v d x d t .
$$

Here $\nabla v:=\left(v_{x_{1}}, \ldots, v_{x_{n}}\right),(\cdot, \cdot)$ is a scalar product in $\mathbb{R}^{n}$.

Note that the integral operator $E: L^{2}\left(Q_{0, T}\right) \rightarrow L^{2}\left(Q_{0, T}\right)$ (see (3) ) is a linear bounded operator and for every $u \in L^{2}\left(Q_{0, T}\right)$ we have

$$
\left\|E u ; L^{2}\left(Q_{0, T}\right)\right\| \leq E^{*}\left\|u ; L^{2}\left(Q_{0, T}\right)\right\|,
$$

where $E^{*}>0$ is independent of $u$. Indeed, using the Cauchy-Bunyakowski-Schwarz inequality (see Lemma 6.1 [27, Chapter $1, \S 6]$ ), we get

$$
\begin{gathered}
\int_{Q_{0, T}}|(E u)(x, t)|^{2} d x d t=\int_{Q_{0, T}}\left|\int_{\Omega} \mathfrak{Z}(x, t, z)(u(x+z, t)-u(x, t)) d z\right|^{2} d x d t \\
\quad \leq \int_{Q_{0, T}}\left(\int_{\Omega}|\mathfrak{Z}(x, t, z)|^{2} d z\right)\left(\int_{\Omega}|u(x+z, t)-u(x, t)|^{2} d z\right) d x d t \\
\leq C_{1} \int_{Q_{0, T}} \int_{\Omega}\left(|u(x+z, t)|^{2}+|u(x, t)|^{2}\right) d z d x d t \leq C_{2} \int_{Q_{0, T}}|u(x, t)|^{2} d x d t
\end{gathered}
$$

and so (16) holds.

Assume that the following condition is satisfied:

$(\mathbf{Z}): a>\phi^{*} E^{*} M_{\Omega}$, where $a$ is defined by (1), $\phi^{*}$ is determined from $(\Phi), E^{*}$ is defined by (16), $M_{\Omega}$ is determined from the Friedrichs inequality (see Lemma 1.26 [27, Chapter $2, \S 1]$ )

$$
\int_{\Omega}|w|^{2} d x \leq M_{\Omega} \int_{\Omega} \sum_{i=1}^{n}\left|w_{x_{i}}\right|^{2} d x, \quad w \in H_{0}^{1}(\Omega) .
$$

Notice that $M_{\Omega}$ depends on $n$ and does not depend on $u$.

Theorem 1. Suppose that $\partial \Omega \in C^{4}$, conditions $(\boldsymbol{A})$-(U) hold, and $(\boldsymbol{Z})$ is satisfied. If $g_{t} \in L^{\infty}\left(Q_{0, T}\right)$ and if $\phi \in L^{\infty}(\mathbb{R})$, then problem (1)-(2) has a weak solution $u$ such that $u \in W^{1, \gamma}\left(Q_{0, T}\right)$ and $|u|^{\frac{r}{2}-1} u \in H^{1}\left(0, T ; L^{2}(\Omega)\right)$.

\section{Auxiliary facts}

\subsection{Functional spaces and some operators}

First we recall some properties of the generalized Lebesgue spases.

Proposition 1. ([37, p. 31]). If $\delta \in \mathscr{B}_{+}(\mathrm{Q})$ and $\delta_{0}>1$, then for every $\eta>0$ there exists a number $Y_{\delta}(\eta)>0$ such that for every $a, b \geq 0$ and for a.e. $y \in Q$ the generalized Young inequality (the Young inequality, if $\delta \equiv$ const)

$$
a b \leq \eta a^{\delta(y)}+Y_{q}(\eta) b^{\delta^{\prime}(y)}
$$


holds. In addition, $Y_{\delta}(\eta)$ depends on $\delta_{0}, \delta^{0}$ and it is independent of $y, Y_{2}(\eta)=\frac{1}{4 \eta}, Y_{2}\left(\frac{1}{2}\right)=\frac{1}{2}, Y_{\delta}(+0)=+\infty$, and $Y_{\delta}(+\infty)=0$.

Proposition 2. Assume that $\delta \in \mathscr{B}_{+}(\mathrm{Q})$ and $\delta_{0}>1$. Then the following statements are fulfilled:

(i) $([30, p .600])$ if $\delta(y) \geq r(y) \geq 1$ for a.e. $y \in \mathrm{Q}$, then $L^{\delta(y)}(\mathrm{Q}) \circlearrowleft L^{r(y)}(\mathrm{Q})$ and

$$
\left\|v ; L^{r(y)}(\mathrm{Q})\right\| \leq(1+\operatorname{mes} \mathrm{Q})\left\|v ; L^{\delta(y)}(\mathrm{Q})\right\|, \quad v \in L^{\delta(y)}(\mathrm{Q})
$$

(ii) ([26, p. 431]) for every $u \in L^{\delta(y)}(\mathrm{Q})$ and $v \in L^{\delta^{\prime}(y)}(\mathrm{Q})$ we get $u v \in L^{1}(\mathrm{Q})$ and the following generalized Hölder inequality is true

$$
\int_{\mathrm{Q}}|u(y) v(y)| d y \leq 2\left\|u ; L^{\delta(y)}(\mathrm{Q})\right\| \cdot\left\|v ; L^{\delta^{\prime}(y)}(\mathrm{Q})\right\| \cdot
$$

Proposition 3. ([11, p. 168]). Suppose that $\delta \in \mathscr{B}_{+}(\mathrm{Q}), \delta_{0}>1, S_{\delta}$ is defined by (8), and $\rho_{\delta}$ is defined by (10). Then for every $v \in \mathscr{M}(\mathrm{Q})$ the following statements are fulfilled:

(i) $\left\|v ; L^{\delta(y)}(\mathrm{Q})\right\| \leq S_{1 / \delta}\left(\rho_{\delta}(v ; \mathrm{Q})\right)$ if $\rho_{\delta}(v ; \mathrm{Q})<+\infty$;

(ii) $\rho_{\delta}(v ; \mathrm{Q}) \leq S_{\delta}\left(\left\|v ; L^{\delta(y)}(\mathrm{Q})\right\|\right)$ if $\left\|v ; L^{\delta(y)}(\mathrm{Q})\right\|<+\infty$.

Proposition 4. (Lemma 1 [7, p. 714]). Suppose that $\delta \in \mathscr{B}_{+}(\mathrm{Q}), \delta_{0}>1$, and $\left\{u^{m}\right\}_{m \in \mathbb{N}} \subset L^{\delta(y)}(\mathrm{Q})$. If $u^{m} \underset{m \rightarrow \infty}{\longrightarrow}$ u weakly in $L^{\delta(y)}(\mathrm{Q})$ and if $u^{m} \underset{m \rightarrow \infty}{\longrightarrow}$ valmost everywhere in $\mathrm{Q}$, then $u=v$.

Let $\Delta^{0} v:=v, \Delta^{1} v:=\Delta v, \Delta^{r} v:=\Delta\left(\Delta^{r-1} v\right)$, and

$$
H_{\Delta}^{2 r}(\Omega):=\left\{v \in H^{2 r}(\Omega)|v|_{\partial \Omega}=\left.\Delta v\right|_{\partial \Omega}=\ldots=\left.\Delta^{r-1} v\right|_{\partial \Omega}=0\right\}, \quad r \in \mathbb{N} .
$$

Take a number $r \in \mathbb{N}$. It is easy to verify that $H_{\Delta}^{2 r}(\Omega)$ is the reflexive Hilbert space with respect to the scalar product

$$
(u, v)_{H_{\Delta}^{2 r}(\Omega)}:=\int_{\Omega} \Delta^{r} u(x) \Delta^{r} v(x) d x, \quad u, v \in H_{\Delta}^{2 r}(\Omega) .
$$

If $\partial \Omega \subset C^{1}$, then the following integration by parts formulae is true

$$
\int_{\Omega} v \Delta^{r} u d x=\int_{\Omega} u \Delta^{r} v d x, \quad u, v \in H_{\Delta}^{2 r}(\Omega) .
$$

Proposition 5. (Lemma 3 [35, p. 229]). If $\partial \Omega \subset C^{2 r}$, then there exists a constant $C_{3}>0$ such that

$$
\left\|v ; H^{2 r}(\Omega)\right\| \leq C_{3}\left\|\Delta^{r} v ; L^{2}(\Omega)\right\|
$$

holds for all $v \in H_{\Delta}^{2 r}(\Omega)$.

Proposition 5 and definition (20) imply that

$$
H_{\Delta}^{2 r}(\Omega) \circlearrowleft H^{2 r}(\Omega), \quad H_{\Delta}^{2 r}(\Omega) \bar{\circlearrowleft} L^{2}(\Omega) \bar{\circlearrowleft}\left[H_{\Delta}^{2 r}(\Omega)\right]^{*} .
$$

Let $\left\{w^{j}\right\}_{j \in \mathbb{N}}$ is an orthonormal basis for the space $L^{2}(\Omega)$ that consists of all eigenfunctions of the problem

$$
-\Delta w=\lambda w \text { in } \Omega,\left.\quad w\right|_{\partial \Omega}=0, \quad w \in H_{0}^{1}(\Omega)
$$


and $\left\{\lambda_{j}\right\}_{j \in \mathbb{N}} \subset \mathbb{R}_{+}$is the set of the corresponding eigenvalues. It is easy to verify that the functions $\left\{w^{j}\right\}_{j \in \mathbb{N}}$ satisfy the equalities

$$
(-1)^{r} \Delta^{r} w^{j}=\lambda_{j}^{r} w^{j} \quad \text { in } \Omega,\left.\quad w^{j}\right|_{\partial \Omega}=\left.\Delta w^{j}\right|_{\partial \Omega}=\ldots=\left.\Delta^{r-1} w^{j}\right|_{\partial \Omega}=0 .
$$

The following statements are needed for the sequel.

Proposition 6. (Theorem 8 [35, p. 230]). If $\partial \Omega \subset C^{2 r}$, then the set $\left\{w^{j}\right\}_{j \in \mathbb{N}}$ is a basis for the space $H_{\Delta}^{2 r}(\Omega)$.

Remark 1 Let us consider the eigenvalue problem

$$
-v_{t t}-v_{x_{1} x_{1}}-v_{x_{2} x_{2}}-\ldots-v_{x_{n} x_{n}}=\zeta v \text { in } Q_{0, T},\left.\quad v\right|_{\partial \Omega \times(0, T)}=0,\left.\quad v\right|_{t=0}=0,\left.\quad v_{t}\right|_{t=T}=0 .
$$

Separating of variables $v(x, t)=w(x) \theta(t)$ yields

$$
\begin{aligned}
&-w(x) \theta^{\prime \prime}(t)-w_{x_{1} x_{1}}(x) \theta(t)-w_{x_{2} x_{2}}(x) \theta(t)-\ldots-w_{x_{n} x_{n}}(x) \theta(t)=\zeta w(x) \theta(t), \\
&-\frac{\theta^{\prime \prime}(t)}{\theta(t)}-\frac{\Delta w(x)}{w(x)}=\zeta,
\end{aligned}
$$

and so $\zeta=\sigma+\lambda, \theta^{\prime \prime}(t)+\sigma \theta(t)=0$ for $t \in(0, T)$, and $\Delta w(x)+\lambda w(x)=0$ for $x \in \Omega$. Hence, instead of $(26)$ we obtain the uncouple system of the following problem:

(i) problem (24);

(ii) the problem

$$
\theta^{\prime \prime}(t)+\sigma \theta(t)=0 \text { for } t \in(0, T), \quad \theta(0)=\theta^{\prime}(T)=0
$$

The eigenvalues and the corresponding eigenfunctions of problem (27) have the following form:

$$
\sigma^{k}=\left(\frac{\pi(2 k-1)}{2 T}\right)^{2}, \quad k \in \mathbb{N}, \quad \theta^{k}(t)=\sin \left(\frac{\pi(2 k-1)}{2 T} t\right), \quad t \in(0, T), \quad k \in \mathbb{N} .
$$

Whence, the eigenvalues and the corresponding eigenfunctions of problem (26) have the following form:

$$
\zeta=\lambda^{j}+\sigma^{k}, \quad v(x, t)=w^{j}(x) \sin \left(\frac{\pi(2 k-1)}{2 T} t\right), \quad(x, t) \in Q_{0, T}, \quad j, k \in \mathbb{N} .
$$

If we renumber it, then we get the set $\left\{v^{m}\right\}_{m \in \mathbb{N}}$ of the eigenfunctions and the set $\left\{\zeta_{m}\right\}_{m \in \mathbb{N}}$ of the corresponding eigenvalues $\zeta_{m}=\widehat{\lambda_{m}}+\widehat{\sigma_{m}}(m \in \mathbb{N})$ of problem (26). Similarly to [42, Section 11.1] we prove that $\left\{v^{m}\right\}_{m \in \mathbb{N}}$ is a complete set in $L^{2}\left(Q_{0, T}\right)$.

For problem (24) we have Proposition 6. In particular, if $r \in \mathbb{N}$ and if the condition $\partial \Omega \in C^{2 r}$ holds, we obtain $\left\{w^{j}\right\}_{j \in \mathbb{N}} \subset H_{\Delta}^{2 r}(\Omega) \subset H^{2 r}(\Omega)$ and so $\left\{v^{m}\right\}_{m \in \mathbb{N}} \subset C^{\infty}\left([0, T] ; H^{2 r}(\Omega)\right) \subset H^{2 r}\left(Q_{0, T}\right)$. Moreover, the positive numbers $\left\{\widehat{\lambda_{m}}\right\}_{m \in \mathbb{N}}$ and $\left\{\widehat{\sigma_{m}}\right\}_{m \in \mathbb{N}}$ satisfy the equalities

$$
\widehat{\lambda_{m}} v^{m}=-\Delta v^{m} \text { in } Q_{0, T}, \quad \widehat{\sigma_{m}} v^{m}=-v_{t t}^{m} \text { in } Q_{0, T}, \quad m \in \mathbb{N}
$$

Lemma 1. If $p \in[1,+\infty)$ and if $z, z_{t} \in L^{p}\left(Q_{0, T}\right)$, then the following inequalities are true:

$$
\int_{\Omega}|z(x, \tau)|^{p} d x \leq C_{4}\left(\int_{\Omega}|z(x, 0)|^{p} d x+\int_{Q_{0, \tau}}\left|z_{t}(x, t)\right|^{p} d x d t\right), \quad \tau \in[0, T],
$$




$$
\begin{gathered}
\int_{\Omega}|z(x, \tau)|^{p} d x \leq C_{4}\left(\int_{\Omega}|z(x, T)|^{p} d x+\int_{Q_{\tau, T}}\left|z_{t}(x, t)\right|^{p} d x d t\right), \quad \tau \in[0, T], \\
\int_{\Omega}|z(x, \tau)|^{p} d x \leq C_{4} \int_{Q_{0, T}}\left[|z(x, t)|^{p}+\left|z_{t}(x, t)\right|^{p}\right] d x d t, \quad \tau \in[0, T],
\end{gathered}
$$

where $C_{4}>0$ is independent of $z, \tau$.

Proof. The proof of estimate (31) is found in Lemma 6 [13]. In the same way we prove (29) and (30).

Lemma 2. Suppose that the Nemytskij operators $G$ and $\mathscr{R}$ are defined by (12) and (13) respectively. If $q, r \in \mathscr{B}_{+}(\Omega)$, $q_{0}>1, r_{0}>1$, and $g \in L^{\infty}\left(Q_{0, T}\right)$, then the operators $G: L^{q(x)}\left(Q_{0, T}\right) \rightarrow L^{q^{\prime}(x)}\left(Q_{0, T}\right)$ and $\mathscr{R}: L^{r(x)}\left(Q_{0, T}\right) \rightarrow L^{r^{\prime}(x)}\left(Q_{0, T}\right)$ are bounded and continuous.

We shall omit the proof because it is analogous to the proof which was given in [30, p. 613].

Proposition 7. (the Aubin theorem, see [3] and [5, p. 393]). If $s, h>1$ are fixed numbers, $\mathscr{W}, \mathscr{L}, \mathscr{B}$ are the Banach spaces, and $\mathscr{W} \stackrel{K}{\subset} \mathscr{L} \circlearrowleft \mathscr{B}$, then $\left\{u \in L^{s}(0, T ; \mathscr{W}) \mid u_{t} \in L^{h}(0, T ; \mathscr{B})\right\} \stackrel{K}{\subset}\left[L^{s}(0, T ; \mathscr{L}) \cap C([0, T] ; \mathscr{B})\right]$.

Proposition 8. (the Vishyk lemma, see Lemma 4.3 [33, p. 66] and [23, p. 60]). Suppose that $m \in \mathbb{N}$, the vector valued function $P:=\left(P_{1}, \ldots, P_{m}\right): \mathbb{R}^{m} \rightarrow \mathbb{R}^{m}$ is continuous, and there exists a number $\rho>0$ such that $(P(z), z)_{\mathbb{R}^{m}} \geq 0$ holds for all $z \in \mathbb{R}^{m}$ such that $|z|=\rho$. Then there exists a vector $z^{m} \in \mathbb{R}^{m}$ such that $\left|z^{m}\right| \leq \rho$ and $P\left(z^{m}\right)=0$.

\subsection{Differentiability of nonlinear expressions}

The following statements are needed for the sequel.

Theorem 2. Suppose that $r \in \mathscr{B}_{+}(\Omega)$. Then the following statements are fulfilled:

(1) If $r_{0}>1$, then the equality

$$
\left(|u|^{r(x)}\right)_{t}=r(x)|u|^{r(x)-2} u u_{t}
$$

is true if one of the following alternatives hold:

(i) $u \in C^{1}\left(\overline{Q_{0, T}}\right)$ (here we have $|u|^{r(x)},\left(|u|^{r(x)}\right)_{t} \in L^{\infty}\left(Q_{0, T}\right)$ );

(ii) $u, u_{t} \in L^{p(x)}\left(Q_{0, T}\right)$ and $p(x) \geq r(x)$ for a.e. $x \in \Omega$ (here we have $|u|^{r(x)},\left(|u|^{r(x)}\right)_{t} \in L^{\frac{p(x)}{r(x)}}\left(Q_{0, T}\right)$ ).

(2) If $r_{0}>2$, then the equality

$$
\left(|u|^{r(x)-2} u\right)_{t}=(r(x)-1)|u|^{r(x)-2} u_{t}
$$

is true if one of the following alternatives hold:

(i) $u \in C^{1}\left(\overline{Q_{0, T}}\right)$ (here we have $|u|^{r(x)-2} u,\left(|u|^{r(x)-2} u\right)_{t} \in L^{\infty}\left(Q_{0, T}\right)$ );

(ii) $u, u_{t} \in L^{p(x)}\left(Q_{0, T}\right)$ and $p(x) \geq r(x)-1$ for a.e. $x \in \Omega$ (here $|u|^{r(x)-2} u,\left(|u|^{r(x)-2} u\right)_{t} \in L^{\frac{p(x)}{r(x)-1}}\left(Q_{0, T}\right)$ ).

This Theorem 2 coincides with Theorem 3 [13] (see also Remark 1 [13]). Then the proof is omitted.

Proposition 9. (Theorem $2\left[25\right.$, p. 286]). If $X$ is the Banach space and if $p \in[1, \infty]$, then $W^{1, p}(0, T ; X) \circlearrowleft C([0, T] ; X)$ and the following integration by parts formulae is true

$$
\int_{s}^{\tau} u_{t}(t) d t=u(\tau)-u(s), \quad 0 \leq s<\tau \leq T, \quad u \in W^{1, p}(0, T ; X) .
$$


Lemma 3. Suppose that $\Omega \subset \mathbb{R}^{n}$ is a bounded $C^{0,1}$-domain (see [27, p. 48]). Then the integration by parts formulae

$$
\int_{Q_{s, \tau}} w_{t} z d x d t=\left.\int_{\Omega_{t}} w z d x\right|_{t=s} ^{t=\tau}-\int_{Q_{s, \tau}} w z_{t} d x d t, \quad 0 \leq s<\tau \leq T
$$

holds if one of the following alternatives hold:

(i) $w \in L^{q(x)}\left(Q_{0, T}\right)$, where $q \in \mathscr{B}_{+}(\Omega), q_{0}>1, w_{t} \in L^{1}\left(Q_{0, T}\right), z \in L^{\infty}\left(Q_{0, T}\right)$, and $z_{t} \in L^{q^{\prime}(x)}\left(Q_{0, T}\right)$;

(ii) $w, w_{t} \in L^{1}\left(Q_{0, T}\right)$ and $z, z_{t} \in L^{\infty}\left(Q_{0, T}\right)$.

Proof. (i). Take $W:=\left\{w \in L^{q(x)}\left(Q_{0, T}\right) \mid w_{t} \in L^{1}\left(Q_{0, T}\right)\right\}$ and $Z:=\left\{z \in L^{\infty}\left(Q_{0, T}\right) \mid z_{t} \in L^{q^{\prime}(x)}\left(Q_{0, T}\right)\right\}$. If $\varphi \in C^{1}([0, T])$ and if $z \in Z$, then $\varphi z \in W^{1,1}\left(0, T ; L^{\frac{q^{0}}{q^{0}-1}}(\Omega)\right)$. By (34) with $u=\varphi(t) z(x, t)$, we get

$$
\int_{s}^{\tau} \varphi_{t}(t) z(x, t) d t=\varphi(\tau) z(x, \tau)-\varphi(s) z(x, s)-\int_{s}^{\tau} \varphi(t) z_{t}(x, t) d t, \quad x \in \Omega .
$$

Take a function $v \in C^{1}(\bar{\Omega})$. By (36), we obtain

$$
\int_{Q_{s, \tau}} \varphi_{t} v z d x d t=\left.\int_{\Omega_{t}} \varphi v z d x\right|_{t=s} ^{t=\tau}-\int_{Q_{s, \tau}} \varphi v z_{t} d x d t
$$

Clearly, $C^{1}\left([0, T] ; C^{1}(\bar{\Omega})\right) \bar{\circlearrowleft} \bar{\circlearrowleft} W^{1,1}\left(0, T ; L^{1}(\Omega)\right)$. Then the set

$$
\left\{\sum_{i=1}^{m} \varphi_{i}(t) v_{i}(x) \mid m \in \mathbb{N}, \quad \varphi_{1}, \ldots, \varphi_{m} \in C^{1}([0, T]), \quad v_{1}, \ldots, v_{m} \in C^{1}(\bar{\Omega})\right\}
$$

is dense in $W$ and (37) yields (35).

We shall omit the proof of (ii) because it is analogous to the previous one.

Lemma 4. Suppose that $\alpha \in \mathscr{B}_{+}(\mathrm{Q})$,

$$
\psi_{\alpha(y)}(s):=\left\{\begin{aligned}
s^{\alpha(y)} & \text { if } s>0, \\
0 & \text { if } s \leq 0,
\end{aligned} \quad y \in \mathrm{Q}\right.
$$

$p, \delta \in \mathscr{B}_{+}(\mathrm{Q}), p_{0}, \delta_{0}>1, p(y) \geq \alpha(y)$ for a.e. $y \in \mathrm{Q}$, and $\delta(y) \leq \frac{p(y)}{\alpha(y)}$ for a.e. $y \in \mathrm{Q}$. Then for every $u \in L^{p(y)}(\mathrm{Q})$ we have that $\psi_{\alpha(y)}(u) \in L^{\frac{p(y)}{\alpha(y)}}(\mathrm{Q})$,

$$
\begin{gathered}
\rho_{p / \alpha}\left(\psi_{\alpha(y)}(u) ; \mathrm{Q}\right) \leq \rho_{p}(u ; \mathrm{Q}), \\
\left\|\psi_{\alpha(y)}(u) ; L^{\delta(y)}(\mathrm{Q})\right\| \leq C_{5} S_{\alpha / p}\left(\rho_{p}(u ; \mathrm{Q})\right),
\end{gathered}
$$

where $C_{5}>0$ is independent of $u$.

Proof. Clearly, $\frac{p(y)}{\alpha(y)} \geq 1$ for a.e. $y \in \mathrm{Q},\left.\left|\psi_{\alpha(y)}(u)^{\frac{p(y)}{\alpha(y)}} \leq\right| u\right|^{p(y)} \in L^{1}(\mathrm{Q})$. Then by [29, p. 297], we obtain $\psi_{\alpha(y)}(u) \in L^{\frac{p(y)}{\alpha(y)}}(\mathrm{Q})$. Moreover, (39) and

$$
\left\|\psi_{\alpha(y)}(u) ; L^{\delta(y)}(\mathrm{Q})\right\| \leq C_{6}\left\|\psi_{\alpha(y)}(u) ; L^{p(y) / \alpha(y)}(\mathrm{Q})\right\| \leq C_{6} S_{\alpha / p}\left(\rho_{p / \alpha}\left(\psi_{\alpha(y)}(u) ; \mathrm{Q}\right)\right)
$$


hold. This inequality and (39) imply (40).

Lemma 5. Suppose that $\beta \in \mathscr{B}_{+}(\Omega), \psi_{\beta(x)}$ is determined from (38) if we replace $\alpha(y)$ by $\beta(x)$, and

$$
\chi_{k}(s):=\left\{\begin{array}{l}
1 \text { if } s>\frac{1}{k}, \\
0 \text { if } s \leq \frac{1}{k},
\end{array} \quad k \in \mathbb{N} .\right.
$$

If $u \in C^{1}\left(\overline{Q_{0, T}}\right)$ and if $v, v_{t} \in L^{1}\left(Q_{0, T}\right)$, then

$$
\lim _{k \rightarrow+\infty} \int_{Q_{0, T}} \chi_{k}(u) \beta(x) \psi_{\beta(x)-1}(u) u_{t} v d x d t=\left.\int_{\Omega_{t}} \psi_{\beta(x)}(u) v d x\right|_{t=0} ^{t=T}-\int_{Q_{0, T}} \psi_{\beta(x)}(u) v_{t} d x d t .
$$

Proof. By definition, put

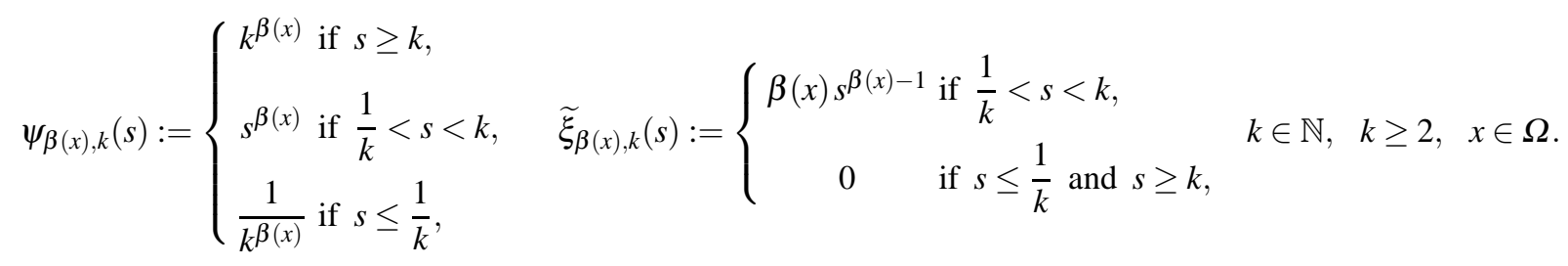

Clearly, $\psi_{\beta(x), k}(s) \underset{k \rightarrow \infty}{\longrightarrow} \psi_{\beta(x)}(s)$ if $s \in \mathbb{R}$ and $x \in \Omega$. In addition, for $k \in \mathbb{N}(k \geq 2)$ and $x \in \Omega$ the function $s \mapsto \psi_{\beta(x), k}(s)$ has the Lipschitz property in $\mathbb{R}$ and it is not differentiable only in the point $s=\frac{1}{k}$ and $s=k$. Moreover, $\frac{\partial}{\partial s} \psi_{\beta(x), k}(s)=\widetilde{\xi}_{\beta(x), k}(s)$ if $s \neq \frac{1}{k}$ and $s \neq k$. Whence, by Lemma 4 [13], we obtain

$$
\left(\psi_{\beta(x), k}(u)\right)_{t}=\widetilde{\xi}_{\beta(x), k}(u) u_{t} \text { almost everywhere in } Q_{0, T} .
$$

Thus, $\psi_{\beta(x), k}(u),\left(\psi_{\beta(x), k}(u)\right)_{t} \in L^{\infty}\left(Q_{0, T}\right)$. Using case (ii) of Lemma 3 with $z=\psi_{\beta(x), k}(u)$ and $w=v$, we get (see (35))

$$
\int_{Q_{0, T}}\left(\psi_{\beta(x), k}(u)\right)_{t} v d x d t=\left.\int_{\Omega} \psi_{\beta(x), k}(u) v d x\right|_{t=0} ^{t=T}-\int_{Q_{0, T}} \psi_{\beta(x), k}(u) v_{t} d x d t .
$$

Let $M:=\max \left\{|u(x, t)|:(x, t) \in \overline{Q_{0, T}}\right\}, k_{0} \in \mathbb{N}, k_{0} \geq \max \{2, M\}$. Since $|u| \leq M \leq k_{0} \leq k$, from (43) we have

$$
\left(\psi_{\beta(x), k}(u)\right)_{t}=\widetilde{\xi}_{\beta(x), k}(u) u_{t}=\chi_{k}(u) \beta(x) \psi_{\beta(x)-1}(u) u_{t},
$$

where $k \geq k_{0}$. By the inequality $\left|\psi_{\beta(x), k}(u(x, t))\right| \leq M^{\beta(x)} \forall(x, t) \in \overline{Q_{0, T}}$ and the Lebesgue Dominate Convergence Theorem, we obtain

$$
\begin{gathered}
\lim _{k \rightarrow+\infty} \int_{\Omega_{t}} \psi_{\beta(x), k}(u) v d x=\int_{\Omega_{t}} \psi_{\beta(x)}(u) v d x \text { if } t=0 \text { and } t=T, \\
\lim _{k \rightarrow+\infty} \int_{Q_{0, T}} \psi_{\beta(x), k}(u) v_{t} d x d t=\int_{Q_{0, T}} \psi_{\beta(x)}(u) v_{t} d x d t .
\end{gathered}
$$

Therefore, (42) follows from (44).

Theorem 3. Suppose that $\alpha \in \mathscr{B}_{+}(\Omega), \frac{1}{2}<\alpha_{0} \leq \alpha(x) \leq \alpha^{0}$ for a.e. $x \in \Omega$, and $\psi_{\alpha(x)}$ is defined by (38) if we replace $\alpha(y)$ by $\alpha(x)$. Then the following statements are fulfilled: 
(1) if $u \in C^{2}\left(\overline{Q_{0, T}}\right)$, then $\psi_{\alpha(x)}(u) \in L^{\infty}\left(Q_{0, T}\right)$, $\left(\psi_{\alpha(x)}(u)\right)_{t} \in L^{2}\left(Q_{0, T}\right)$, and

$$
\left(\psi_{\alpha(x)}(u)\right)_{t}=\alpha(x) \psi_{\alpha(x)-1}(u) u_{t}
$$

(2) if $u, u_{t}, u_{t t} \in L^{p(x)}\left(Q_{0, T}\right), p \in L_{+}^{\infty}(\Omega)$, and $p(x) \geq 2 \alpha(x)$ for a.e. $x \in \Omega$, then $\psi_{\alpha(x)}(u) \in W^{1,2}\left(0, T ; L^{2}(\Omega)\right)$, formulae (45) holds, and

$$
\begin{gathered}
\left\|\psi_{\alpha(x)}(u) ; L^{2}\left(Q_{0, T}\right)\right\| \leq C_{7} S_{\alpha / p}\left(\rho_{p}\left(u ; Q_{0, T}\right)\right), \\
\left\|\left(\psi_{\alpha(x)}(u)\right)_{t} ; L^{2}\left(Q_{0, T}\right)\right\| \leq C_{8} S_{\alpha / p}\left(\rho_{p}\left(u ; Q_{0, T}\right)+\rho_{p}\left(u_{t} ; Q_{0, T}\right)+\rho_{p}\left(u_{t t} ; Q_{0, T}\right)\right),
\end{gathered}
$$

where $C_{7}, C_{8}>0$ are independent of $u$.

Proof. First let us prove Case 1. Take a function $u \in C^{2}\left(\overline{Q_{0, T}}\right)$. Since $u_{t}, u_{t t} \in L^{1}\left(Q_{0, T}\right)$, using (42) with $\beta=2 \alpha-1>0$ and $v=u_{t}$, we obtain

$$
\lim _{k \rightarrow+\infty} \int_{Q_{0, T}} f_{k} d x d t=\left.\int_{\Omega_{t}} \psi_{2 \alpha(x)-1}(u) u_{t} d x\right|_{t=0} ^{t=T}-\int_{Q_{0, T}} \psi_{2 \alpha(x)-1}(u) u_{t t} d x d t
$$

where $\chi_{k}$ is defined by $(41), f_{k}=\chi_{k}(u)(2 \alpha(x)-1) \psi_{2 \alpha(x)-2}(u)\left|u_{t}\right|^{2}, \quad k \in \mathbb{N}$. Clearly,

$$
f_{k}(x, t) \underset{k \rightarrow \infty}{\longrightarrow} f(x, t) \text { if }(x, t) \in Q_{0, T},
$$

where $f=(2 \alpha(x)-1) \psi_{2 \alpha(x)-2}(u)\left|u_{t}\right|^{2}=(2 \alpha(x)-1)\left|\psi_{\alpha(x)-1}(u) u_{t}\right|^{2}$. The existence of the limit in (48) implies that there exists a constant $C_{9}>0$ such that for every $k \in \mathbb{N}$ we have the estimate

$$
\int_{Q_{0, T}} f_{k} d x d t \leq C_{9}
$$

Clearly, $f_{k_{1}} \leq f_{k_{2}}$ if $k_{1} \leq k_{2}$. Then the Levi Monotone Convergence Theorem (see Theorem 4.1 [8, p. 90]) and (49) yield that $f \in L^{1}\left(Q_{0, T}\right)$ and so $\psi_{\alpha(x)-1}(u) u_{t} \in L^{2}\left(Q_{0, T}\right)$. In addition,

$$
\int_{Q_{0, T}} f_{k} d x d t \underset{k \rightarrow \infty}{\longrightarrow} \int_{Q_{0, T}} f d x d t
$$

Hence, by (48), we get

$$
\int_{Q_{0, T}}(2 \alpha(x)-1)\left|\psi_{\alpha(x)-1}(u) u_{t}\right|^{2} d x d t=\left.\int_{\Omega_{t}} \psi_{2 \alpha(x)-1}(u) u_{t} d x\right|_{t=0} ^{t=T}-\int_{Q_{0, T}} \psi_{2 \alpha(x)-1}(u) u_{t t} d x d t .
$$

By (50), we obtain

$$
\left\|\chi_{k}(u) \sqrt{2 \alpha-1} \psi_{\alpha(x)-1}(u) u_{t} ; L^{2}\left(Q_{0, T}\right)\right\| \underset{k \rightarrow \infty}{\longrightarrow}\left\|\sqrt{2 \alpha-1} \psi_{\alpha(x)-1}(u) u_{t} ; L^{2}\left(Q_{0, T}\right)\right\| .
$$

Whence, using (49), we prove the following convergence

$$
\chi_{k}(u) \psi_{\alpha(x)-1}(u) u_{t} \underset{k \rightarrow \infty}{\longrightarrow} \psi_{\alpha(x)-1}(u) u_{t} \text { strongly in } L^{2}\left(Q_{0, T}\right)
$$


Take $v \in L^{2}\left(Q_{0, T}\right)$ such that $v_{t} \in L^{1}\left(Q_{0, T}\right)$. Using (53) and (42) with $\beta=\alpha$, we obtain

$$
\int_{Q_{0, T}} \alpha(x) \psi_{\alpha(x)-1}(u) u_{t} v d x d t=\left.\int_{\Omega_{t}} \psi_{\alpha(x)}(u) v d x\right|_{t=0} ^{t=T}-\int_{Q_{0, T}} \psi_{\alpha(x)}(u) v_{t} d x d t
$$

Taking in (54) function $v \in C_{0}^{\infty}\left(Q_{0, T}\right)$, we get

$$
\int_{Q_{0, T}} \alpha(x) \psi_{\alpha(x)-1}(u) u_{t} v d x d t=-\int_{Q_{0, T}} \psi_{\alpha(x)}(u) v_{t} d x d t .
$$

Therefore, (45) holds. Thus, $\left(\psi_{\alpha(x)}(u)\right)_{t} \in L^{2}\left(Q_{0, T}\right)$. Moreover, $\psi_{\alpha(x)}(u) \in L^{\infty}\left(Q_{0, T}\right)$.

Further let us prove Case 2. We start from proof of (46) and (47) if $u \in C^{2}\left(\overline{Q_{0, T}}\right)$. Since $p \geq 2 \alpha$ (and so $2 \leq \frac{p}{\alpha}$ ), estimate (40) with $\mathrm{Q}=Q_{0, T}$ and $q \equiv 2$ yields that (46) holds. Using (45) (we already proved it), by (51), we obtain

$$
\int_{Q_{0, T}}\left|\left(\psi_{\alpha(x)}(u)\right)_{t}\right|^{2} d x d t \leq \frac{\left|\alpha^{0}\right|^{2}}{2 \alpha_{0}-1}\left(\int_{\Omega_{0}} \psi_{2 \alpha(x)-1}(u)\left|u_{t}\right| d x+\int_{\Omega_{T}} \psi_{2 \alpha(x)-1}(u)\left|u_{t}\right| d x+\int_{Q_{0, T}} \psi_{2 \alpha(x)-1}(u)\left|u_{t t}\right| d x d t\right) .
$$

On the other hand, for every $r \in \mathscr{B}_{+}(\Omega)\left(r_{0}>1\right)$ the estimate

$$
J_{1}:=\int_{\Omega_{0}} \psi_{2 \alpha(x)-1}(u)\left|u_{t}\right| d x \leq\left\|\psi_{2 \alpha(x)-1}(u(0)) ; L^{r^{\prime}(x)}(\Omega)\right\| \cdot\left\|u_{t}(0) ; L^{r(x)}(\Omega)\right\|
$$

holds. Since $p \geq 2 \alpha>1$, for $r=2 \alpha$ the following inequalities are true: $1<r \leq p, 2 \alpha-1<p$, and $1<r^{\prime}=\frac{r}{r-1}=\frac{r}{2 \alpha-1} \leq$ $\frac{p}{2 \alpha-1}$. Then estimate (40) with $\mathrm{Q}=\Omega$ and $q=r^{\prime}$ implies that

$$
\left\|\psi_{2 \alpha(x)-1}(u(0)) ; L^{r^{\prime}(x)}(\Omega)\right\| \leq C_{10} S_{(2 \alpha-1) / p}\left(\rho_{p}(u(0) ; \Omega)\right)
$$

holds. In addition, by Proposition 3, we obtain

$$
\left\|u_{t}(0) ; L^{r(x)}(\Omega)\right\| \leq C_{11}\left\|u_{t}(0) ; L^{p(x)}(\Omega)\right\| \leq C_{11} S_{1 / p}\left(\rho_{p}\left(u_{t}(0) ; \Omega\right)\right) .
$$

Therefore, by (31) with $p=p(x)$ and by the equality $S_{a}(z) S_{b}(z)=S_{a+b}(z)(a, b, z \geq 0)$, we get

$$
\begin{gathered}
J_{1} \leq C_{12} S_{(2 \alpha-1) / p}\left(\int_{\Omega}|u(0)|^{p(x)} d x\right) S_{1 / p}\left(\int_{\Omega}\left|u_{t}(0)\right|^{p(x)} d x\right) \\
\leq C_{13} S_{2 \alpha / p}\left(\int_{Q_{0, T}}\left[|u(x, t)|^{p(x)}+\left|u_{t}(x, t)\right|^{p(x)}+\left|u_{t t}(x, t)\right|^{p(x)}\right] d x d t\right),
\end{gathered}
$$

where $C_{13}>0$ is independent of $u$. Continuing in the same way, by (55), we obtain (47).

Finally let us take $u, u_{t}, u_{t t} \in L^{p(x)}\left(Q_{0, T}\right), p \geq 2 \alpha$, and $\left\{u^{\ell}\right\}_{\ell \in \mathbb{N}} \subset C^{2}\left(\overline{Q_{0, T}}\right)$, where

$$
u^{\ell} \underset{\ell \rightarrow \infty}{\longrightarrow} u, \quad u_{t}^{\ell} \underset{\ell \rightarrow \infty}{\longrightarrow} u_{t}, \quad u_{t t}^{\ell} \underset{\ell \rightarrow \infty}{\longrightarrow} u_{t t} \text { strongly in } L^{p(x)}\left(Q_{0, T}\right) .
$$


Estimates (46) and (47) imply that the sequence $\left\{\psi_{\alpha(x)}\left(u^{\ell}\right)\right\}_{\ell \in \mathbb{N}}$ is bounded in $W^{1,2}\left(0, T ; L^{2}(\Omega)\right)$. Then there exists a subsequence $\left\{u^{\ell_{k}}\right\}_{k \in \mathbb{N}} \subset\left\{u^{\ell}\right\}_{\ell \in \mathbb{N}}$ such that

$$
\psi_{\alpha(x)}\left(u^{\ell_{k}}\right) \underset{k \rightarrow \infty}{\longrightarrow} \chi \text { weakly in } W^{1,2}\left(0, T ; L^{2}(\Omega)\right)
$$

and so $\alpha \psi_{\alpha(x)-1}\left(u^{\ell_{k}}\right) u_{t}^{\ell_{k}}=\left(\psi_{\alpha(x)}\left(u^{\ell_{k}}\right)\right)_{t} \underset{k \rightarrow \infty}{\longrightarrow} \zeta$ weakly in $L^{2}\left(Q_{0, T}\right)$. By choosing the sequence $\left\{u^{\ell}\right\}_{\ell \in \mathbb{N}}$, there exists a subsequence (we call it $\left\{u^{\ell_{k}}\right\}_{k \in \mathbb{N}}$ again) such that

$$
\begin{gathered}
u^{\ell_{k}} \underset{k \rightarrow \infty}{\longrightarrow} u, \quad u_{t}^{\ell_{k}} \underset{k \rightarrow \infty}{\longrightarrow} u_{t} \text { almost everywhere in } Q_{0, T} \\
\alpha(x) \psi_{\alpha(x)-1}\left(u^{\ell_{k}}\right) u_{t}^{\ell_{k}} \underset{k \rightarrow \infty}{\longrightarrow} \alpha(x) \psi_{\alpha(x)-1}(u) u_{t} \text { almost everywhere in } Q_{0, T} .
\end{gathered}
$$

Therefore $\chi=\psi_{\alpha(x)}(u)$ and $\zeta=\alpha \psi_{\alpha(x)-1}(u) u_{t}$. Thus, (54) with $v \in W^{1,2}\left(0, T ; L^{2}(\Omega)\right)$ holds and so (45) is true.

Since $\left\{u^{\ell}\right\}_{\ell \in \mathbb{N}} \subset C^{2}\left(\overline{Q_{0, T}}\right)$ inequality (46) and (47) with $u=u^{\ell}$ are true, i.e.

$$
\begin{gathered}
\left\|\psi_{\alpha(x)}\left(u^{\ell_{k}}\right) ; L^{2}\left(Q_{0, T}\right)\right\| \leq C_{7} S_{\alpha / p}\left(\rho_{p}\left(u^{\ell_{k}} ; Q_{0, T}\right)\right), \\
\left\|\left(\psi_{\alpha(x)}\left(u^{\ell_{k}}\right)\right)_{t} ; L^{2}\left(Q_{0, T}\right)\right\| \leq C_{8} S_{\alpha / p}\left(\rho_{p}\left(u^{\ell_{k}} ; Q_{0, T}\right)+\rho_{p}\left(u_{t}^{\ell_{k}} ; Q_{0, T}\right)+\rho_{p}\left(u_{t t}^{\ell_{k}} ; Q_{0, T}\right)\right),
\end{gathered}
$$

where $C_{7}, C_{8}>0$ are independent of $u^{\ell_{k}}, \ell_{k}$. Thus, using Lemma 5.3 [27, p. 20], we obtain (46) and (47).

Notice that the case $\alpha(x) \in\left(0, \frac{1}{2}\right]$ (see Theorem 3) is considered in [12].

Theorem 4. Assume that $r \in \mathscr{B}_{+}(\Omega)$. Then the following statements are fulfilled:

(1) If $\frac{1}{2}<r_{0} \leq r^{0} \leq 1$, then the equality (32) is true if one of the following alternatives hold:

(i) $u \in C^{2}\left(\overline{Q_{0, T}}\right)$ (here we have $|u|^{r(x)} \in L^{\infty}\left(Q_{0, T}\right)$ and $\left(|u|^{r(x)}\right)_{t} \in L^{2}\left(Q_{0, T}\right)$ );

(ii) $u, u_{t}, u_{t t} \in L^{p(x)}\left(Q_{0, T}\right)$ and $p(x) \geq 2 r(x)$ for a.e. $x \in \Omega$ (here we have $|u|^{r(x)} \in H^{1}\left(0, T ; L^{2}(\Omega)\right)$ and

$$
\left\||u|^{r(x)} ; H^{1}\left(0, T ; L^{2}(\Omega)\right)\right\| \leq C_{14} S_{r / p}\left(\rho_{p}\left(u ; Q_{0, T}\right)+\rho_{p}\left(u_{t} ; Q_{0, T}\right)+\rho_{p}\left(u_{t t} ; Q_{0, T}\right)\right),
$$

where $C_{14}>0$ is independent of $u$ ).

(2) If $\frac{3}{2}<r_{0} \leq r^{0} \leq 2$, then the equality (33) is true if one of the following alternatives hold:

(i) $u \in C^{2}\left(\overline{Q_{0, T}}\right)$ (here we have $|u|^{r(x)-2} u \in L^{\infty}\left(Q_{0, T}\right)$ and $\left(|u|^{r(x)-2} u\right)_{t} \in L^{2}\left(Q_{0, T}\right)$ );

(ii) $u, u_{t}, u_{t t} \in L^{p(x)}\left(Q_{0, T}\right)$ and $p(x) \geq 2(r(x)-1)$ for a.e. $x \in \Omega$ (here we have $|u|^{r(x)-2} u \in H^{1}\left(0, T ; L^{2}(\Omega)\right)$ and

$$
\left\||u|^{r(x)-2} u ; H^{1}\left(0, T ; L^{2}(\Omega)\right)\right\| \leq C_{15} S_{(r-1) / p}\left(\rho_{p}\left(u ; Q_{0, T}\right)+\rho_{p}\left(u_{t} ; Q_{0, T}\right)+\rho_{p}\left(u_{t t} ; Q_{0, T}\right)\right),
$$

where $C_{15}>0$ is independent of $u$.

Proof. The proof of Theorem 4 follows from Theorem 3 if we recall that

$$
|s|^{r(x)}=\psi_{r(x)}(s)+\psi_{r(x)}(-s), \quad|s|^{r(x)-2} s=\psi_{r(x)-1}(s)-\psi_{r(x)-1}(-s), \quad x \in \Omega, \quad s \in \mathbb{R} .
$$

Notice that we omit the notation $\psi$ in the right-hand sides of (32) and (33). Thus the right-hand sides of (32) and (33) equal zero on $\left\{(x, t) \in Q_{0, T} \mid u(x, t)=0\right\}$.

If we take $p(x) \equiv$ const and $r(x) \equiv$ const, we rewrite Theorem 4 as follows. 
Theorem 5. Assume that $r>0$. Then the following statements are fulfilled:

(1) If $\frac{1}{2}<r \leq 1$, then the equality

$$
\left(|u|^{r}\right)_{t}=r|u|^{r-2} u u_{t}
$$

is true if one of the following alternatives hold:

(i) $u \in C^{2}\left(\overline{Q_{0, T}}\right)$ (here we have $|u|^{r} \in C\left(\overline{Q_{0, T}}\right)$ and $\left(|u|^{r}\right)_{t} \in L^{2}\left(Q_{0, T}\right)$ );

(ii) $u \in W^{2, p}\left(0, T ; L^{p}(\Omega)\right)$ and $p \geq 2 r$ (here we have $|u|^{r} \in H^{1}\left(0, T ; L^{2}(\Omega)\right)$ and

$$
\left\|\left.|| u\right|^{r} ; H^{1}\left(0, T ; L^{2}(\Omega)\right)\right\| \leq C_{16}\left(\left\|u ; L^{p}\left(Q_{0, T}\right)\right\|^{r}+\left\|u_{t} ; L^{p}\left(Q_{0, T}\right)\right\|^{r}+\left\|u_{t t} ; L^{p}\left(Q_{0, T}\right)\right\|^{r}\right),
$$

where $C_{16}>0$ is independent of $u$.

(2) If $\frac{3}{2}<r \leq 2$, then the equality

$$
\left(|u|^{r-2} u\right)_{t}=(r-1)|u|^{r-2} u_{t}
$$

is true if one of the following alternatives hold:

(i) $u \in C^{2}\left(\overline{Q_{0, T}}\right)$ (here we have $|u|^{r-2} u \in C\left(\overline{Q_{0, T}}\right)$ and $\left(|u|^{r-2} u\right)_{t} \in L^{2}\left(Q_{0, T}\right)$ );

(ii) $u \in W^{2, p}\left(0, T ; L^{p}(\Omega)\right)$ and $p \geq 2(r-1)$ (here we have $|u|^{r-2} u \in H^{1}\left(0, T ; L^{2}(\Omega)\right.$ ) and

$$
\left\||u|^{r-2} u ; H^{1}\left(0, T ; L^{2}(\Omega)\right)\right\| \leq C_{17}\left(\left\|u ; L^{p}\left(Q_{0, T}\right)\right\|^{r-1}+\left\|u_{t} ; L^{p}\left(Q_{0, T}\right)\right\|^{r-1}+\left\|u_{t t} ; L^{p}\left(Q_{0, T}\right)\right\|^{r-1}\right)
$$

where $C_{17}>0$ is independent of $u$.

\subsection{Boundary value problem for some elliptic equation}

The following Dirichlet-Neumann boundary value problem is needed for the sequel

$$
\begin{gathered}
-\varepsilon u_{t t}^{\varepsilon}+\left(\mathscr{R} u^{\varepsilon}\right)_{t}-a \Delta u^{\varepsilon}+G u^{\varepsilon}+\phi\left(E u^{\varepsilon}\right)=f(x, t), \quad(x, t) \in Q_{0, T}, \\
\left.u^{\varepsilon}\right|_{\Sigma_{0, T}}=0,\left.\quad u^{\varepsilon}\right|_{t=0}=u_{0},\left.\quad u_{t}^{\varepsilon}\right|_{t=T}=0,
\end{gathered}
$$

where $\varepsilon>0$. By definition, put $U_{0}\left(Q_{0, T}\right):=\left\{v \in H^{1}\left(Q_{0, T}\right)|v|_{\Sigma_{0, T}}=0,\left.v\right|_{t=0}=0\right\}$,

$$
D(x, t):=\left(1-\frac{t}{T}\right)^{2} u_{0}(x), \quad(x, t) \in Q_{0, T}
$$

Clearly, (64) and condition (U) yield that $D \in H^{2}\left(Q_{0, T}\right)$,

$$
\left.D\right|_{\Sigma_{0, T}}=0,\left.\quad D\right|_{t=0}=u_{0},\left.\quad D\right|_{t=T}=0,\left.\quad D_{t}\right|_{t=T}=0
$$

Definition 2. A real-valued function $u^{\varepsilon} \in H^{1}\left(Q_{0, T}\right)$ is called a weak solution of problem (62)-(63) if u satisfies (63 1$)$, (632) (i.e. $u^{\varepsilon}-D \in U_{0}\left(Q_{0, T}\right)$, where $D$ is defined by (64) ), $\mathscr{R} u^{\varepsilon}, G u^{\varepsilon}, E u^{\varepsilon} \in L^{2}\left(Q_{0, T}\right)$, and for every $v \in U_{0}\left(Q_{0, T}\right)$ we have

$$
\int_{Q_{0, T}}\left[\varepsilon u_{t}^{\varepsilon} v_{t}-\mathscr{R} u^{\varepsilon} v_{t}+a\left(\nabla u^{\varepsilon}, \nabla v\right)+G u^{\varepsilon} v+\phi\left(E u^{\varepsilon}\right) v\right] d x d t+\int_{\Omega} \mathscr{R} u^{\varepsilon}(T) v(T) d x=\int_{Q_{0, T}} f v d x d t
$$


For functions $w, v: Q_{0, T} \rightarrow \mathbb{R}^{1}$ by definition, put

$$
\mathscr{L}_{\varepsilon}(w, v):=\int_{Q_{0, T}}\left[\varepsilon w_{t} v_{t}+|w|^{r-2} w_{t} v+a(\nabla w, \nabla v)+G w v+\phi(E w) v\right] d x d t
$$

Lemma 6. If $r \in(1,2)$, conditions $(\boldsymbol{G})-(\Phi)$ hold, and $(\boldsymbol{Z})$ is satisfied, then there exists a constant $\alpha_{0}>0$ such that for every $u \in H^{1}\left(Q_{0, T}\right)$ and $\varepsilon>0$ the estimate

$$
\mathscr{L}_{\varepsilon}(u, u) \geq\left.\frac{1}{r} \int_{\Omega}|u(x, t)|^{r} d x\right|_{t=0} ^{t=T}+\int_{Q_{0, T}}\left[\varepsilon\left|u_{t}\right|^{2}+\alpha_{0}\left(|\nabla u|^{2}+|u|^{2}+|u|^{r}\right)+g_{0}|u|^{q(x)}\right] d x d t-C_{18}
$$

holds, where $C_{18}>0$ is independent of $u, \varepsilon$.

Proof. By (67), it follows that

$$
\mathscr{L}_{\varepsilon}(u, u)=\int_{Q_{0, T}}\left[\varepsilon\left|u_{t}\right|^{2}+|u|^{r-2} u_{t} u+a|\nabla u|^{2}+g|u|^{q(x)}+\phi(E u) u\right] d x d t
$$

Since $u \in H^{1}\left(Q_{0, T}\right)$ and $2 \geq r>1$, Case 1.ii of Theorem 2 with $p(x) \equiv 2$ and $r(x) \equiv r$ (see also Proposition 9) implies that

$$
|u|^{r} \in W^{1, \frac{2}{r}}\left(0, T ; L^{\frac{2}{r}}(\Omega)\right), \quad\left(|u|^{r}\right)_{t}=r|u|^{r-2} u u_{t}, \quad \int_{Q_{0, T}}\left(|u|^{r}\right)_{t} d x d t=\left.\int_{\Omega}|u(x, t)|^{r} d x\right|_{t=0} ^{t=T} .
$$

Taking into account condition $(\Phi)$, the Cauchy-Bunyakowski-Schwarz inequality, (16), and (17), we obtain

$$
\left|\int_{Q_{0, T}} \phi(E u) u d x d t\right| \leq \phi^{*}|| E u ; L^{2}\left(Q_{0, T}\right)\|\cdot\| u ; L^{2}\left(Q_{0, T}\right)\left\|\leq \phi^{*} E^{*}\right\| u ; L^{2}\left(Q_{0, T}\right) \|^{2} \leq \phi^{*} E^{*} M_{\Omega} \int_{Q_{0, T}}|\nabla u|^{2} d x d t .
$$

Using the Young inequality with the exponents $\frac{2}{r}, \frac{2}{2-r}>1$ and inequality (17), we get

$$
\int_{\Omega}|u(t)|^{r} d x \leq \int_{\Omega}|u(t)|^{2} d x+C_{19} \leq M_{\Omega} \int_{\Omega}|\nabla u(t)|^{2} d x+C_{19}, \quad t \in[0, T],
$$

where $C_{19}>0$ is independent of $u, t$. Then for $\alpha:=a-\phi^{*} E^{*} M_{\Omega}>0$ we have

$$
\begin{gathered}
\int_{Q_{0, T}}\left[a|\nabla u|^{2}+\phi(E u) u\right] d x d t \geq \alpha \int_{Q_{0, T}}|\nabla u|^{2} d x d t=\int_{Q_{0, T}}\left(\frac{\alpha}{3}+\frac{\alpha}{3}+\frac{\alpha}{3}\right)|\nabla u|^{2} d x d t \geq \\
\geq \int_{Q_{0, T}}\left[\frac{\alpha}{3}|\nabla u|^{2}+\frac{\alpha}{3 M_{\Omega}}|u|^{2}+\frac{\alpha}{3 M_{\Omega}}|u|^{r}\right] d x d t-C_{20} .
\end{gathered}
$$

Taking into account (69) and the transformations above, we obtain (68).

Theorem 6. Suppose that $\partial \Omega \in C^{4}$ and the following conditions are satisfied:

(A): $a>0, \frac{3}{2}<r \leq 2$

$\left(\right.$ Q): $q \in \mathscr{B}_{+}(\Omega), 1<q_{0} \leq q^{0} \leq 2$;

$(\boldsymbol{G}): g \in \mathscr{B}_{+}\left(Q_{0, T}\right)$;

(E): $\mathfrak{Z} \in L^{\infty}\left(Q_{0, T} \times \Omega\right)$;

$(\Phi): \phi \in \operatorname{Lip}(\mathbb{R}),|\phi(\xi)| \leq \phi^{*}|\xi|$ for every $\xi \in \mathbb{R}$, where $\phi^{*} \in[0,+\infty)$;

(F): $f \in L^{r^{\prime}}\left(Q_{0, T}\right)$, where $\frac{1}{r}+\frac{1}{r^{\prime}}=1$; 
$(\boldsymbol{U}): u_{0} \in H^{2}(\Omega) \cap H_{0}^{1}(\Omega)$;

(Z): $a>\phi^{*} E^{*} M_{\Omega}$, where $E^{*}$ is defined by (16), $M_{\Omega}$ is determined from (17).

Then problem (62)-(63) has a weak solution $u^{\varepsilon}$ such that $u^{\varepsilon} \in H^{2}\left(Q_{0, T}\right) \cap H^{1}\left(0, T ; H_{0}^{1}(\Omega)\right)$ and $\mathscr{R} u^{\varepsilon} \in H^{1}\left(0, T ; L^{2}(\Omega)\right)$.

Proof. The Case $r=2$ is trivial. Let us assume that $r \in\left(\frac{3}{2}, 2\right)$. The solution will be constructed via Galerkin's method.

Step 1. Let $\left\{v^{m}\right\}_{m \in \mathbb{N}}$ be determined from Remark 1,D defined by (64), $\varepsilon>0$ a fixed number, $\mathscr{L}_{\varepsilon}$ defined by (67),

$$
u^{\varepsilon, m}(x, t):=D(x, t)+z^{\varepsilon, m}(x, t), \quad z^{\varepsilon, m}(x, t):=\sum_{\mu=1}^{m} \varphi_{\mu}^{\varepsilon, m} v^{\mu}(x, t), \quad(x, t) \in Q_{0, T}, \quad m \in \mathbb{N},
$$

and $\varphi:=\left(\varphi_{1}^{\varepsilon, m}, \ldots, \varphi_{m}^{\varepsilon, m}\right) \in \mathbb{R}^{m}$. For the sake of convenience we have omitted index $\varepsilon$ in $u^{\varepsilon, m}, \varphi_{1}^{\varepsilon, m}, \ldots, \varphi_{m}^{\varepsilon, m}$, and $z^{\varepsilon, m}$. Notice also that

$$
\begin{array}{cc}
\left.z^{m}\right|_{\Sigma_{0, T}}=0,\left.\quad z^{m}\right|_{t=0}=0,\left.\quad z_{t}^{m}\right|_{t=T}=0, \\
\left.u^{m}\right|_{\Sigma_{0, T}}=0,\left.\quad u^{m}\right|_{t=0}=u_{0},\left.\quad u_{t}^{m}\right|_{t=T}=0 .
\end{array}
$$

Since $r<2$, we have $2 \geq 2(r-1)$. In addition, the inequality $r>\frac{3}{2}$ holds. Since $\partial \Omega \in C^{2}$, Remark 1 implies that $\left\{v^{m}\right\}_{m \in \mathbb{N}} \subset H^{2}\left(Q_{0, T}\right)$. Then $\left\{z^{m}\right\}_{m \in \mathbb{N}},\left\{u^{m}\right\}_{m \in \mathbb{N}} \subset H^{2}\left(Q_{0, T}\right)$ and Case 2.ii of Theorem 5 with $p=2$ yields that

$$
\mathscr{R} u^{m} \in H^{1}\left(0, T ; L^{2}(\Omega)\right), \quad\left(\mathscr{R} u^{m}\right)_{t}=\left|u^{m}\right|^{r-2} u_{t}^{m} .
$$

Assume that the unknown vector $\varphi$ (see (71) ) satisfies the following equalities:

$$
\int_{Q_{0, T}}\left[\varepsilon u_{t}^{m} v_{t}^{\mu}+\left|u^{m}\right|^{r-2} u_{t}^{m} v^{\mu}+a\left(\nabla u^{m}, \nabla v^{\mu}\right)+G u^{m} v^{\mu}+\phi\left(E u^{m}\right) v^{\mu}\right] d x d t=\int_{Q_{0, T}} f v^{\mu} d x d t,
$$

where $\mu=\overline{1, m}$. Let us prove that this $\varphi$ exists. Equality (75) we rewrite to read

$$
P(\varphi)=0,
$$

where $P:=\left(P_{1}, \ldots, P_{m}\right), P_{\mu}(\varphi):=\mathscr{L}_{\varepsilon}\left(u^{m}, \nu^{\mu}\right)-\int_{Q_{0, T}} f v^{\mu} d x d t, \mu=\overline{1, m}$. Clearly,

$$
\begin{gathered}
(P(\varphi), \varphi)_{\mathbb{R}^{m}}=\sum_{\mu=1}^{m}\left(\mathscr{L}_{\varepsilon}\left(u^{m}, v^{\mu}\right)-\int_{Q_{0, T}} f v^{\mu} d x d t\right) \varphi_{\mu}^{m}=\mathscr{L}_{\varepsilon}\left(u^{m}, z^{m}\right)-\int_{Q_{0, T}} f z^{m} d x d t \\
=\mathscr{L}_{\varepsilon}\left(u^{m}, u^{m}-D\right)-\int_{Q_{0, T}} f\left(u^{m}-D\right) d x d t=\int_{Q_{0, T}} f D d x d t-\int_{Q_{0, T}} f u^{m} d x d t+\mathscr{L}_{\varepsilon}\left(u^{m}, u^{m}\right)-\mathscr{L}_{\varepsilon}\left(u^{m}, D\right) .
\end{gathered}
$$

Using Young's inequality, we obtain

$$
|f D| \leq \frac{1}{2}|f|^{2}+\frac{1}{2}|D|^{2}, \quad\left|f u^{m}\right| \leq \varkappa_{1}\left|u^{m}\right|^{2}+\frac{1}{4 \varkappa_{1}}|f|^{2}, \quad \varkappa_{1}>0 .
$$

By (68) and $\left(73_{2}\right)$, we get

$$
\mathscr{L}_{\varepsilon}\left(u^{m}, u^{m}\right) \geq \frac{1}{r} \int_{\Omega}\left|u^{m}(T)\right|^{r} d x+\int_{Q_{0, T}}\left[\varepsilon\left|u_{t}^{m}\right|^{2}+\alpha_{0}\left|\nabla u^{m}\right|^{2}+\alpha_{0}\left|u^{m}\right|^{2}+\alpha_{0}\left|u^{m}\right|^{r}+g_{0}\left|u^{m}\right| q(x)\right] d x d t-\frac{1}{r} \int_{\Omega}\left|u_{0}\right|^{r} d x-C_{21},
$$


where $C_{21}>0$ is independent of $m, \varepsilon$.

Now let us consider the expression

$$
\mathscr{L}_{\varepsilon}\left(u^{m}, D\right)=\int_{Q_{0, T}}\left[\varepsilon u_{t}^{m} D_{t}+\left|u^{m}\right|^{r-2} u_{t}^{m} D+a\left(\nabla u^{m}, \nabla D\right)+G u^{m} D+\phi\left(E u^{m}\right) D\right] d x d t .
$$

Using Young's inequality, we obtain

$$
\left|u_{t}^{m} D_{t}\right| \leq \frac{1}{2}\left|u_{t}^{m}\right|^{2}+\frac{1}{2}\left|D_{t}\right|^{2}, \quad\left|a\left(\nabla u^{m}, \nabla D\right)\right| \leq a \varkappa_{2}\left|\nabla u^{m}\right|^{2}+\frac{a}{4 \varkappa_{2}}|\nabla D|^{2}, \quad \varkappa_{2}>0 .
$$

Taking into account (74), integration by parts formulae, (65), (73), and the Young inequality with $r^{\prime}, r>1$, we obtain

$$
\begin{gathered}
\int_{Q_{0, T}}\left|u^{m}\right|^{r-2} u_{t}^{m} D d x d t=\int_{Q_{0, T}}\left(\mathscr{R} u^{m}\right)_{t} D d x d t=\left.\int_{\Omega} \mathscr{R} u^{m} D d x\right|_{t=0} ^{t=T}-\int_{Q_{0, T}} \mathscr{R} u^{m} D_{t} d x d t \\
\leq-\int_{\Omega} \mathscr{R} u_{0} u_{0} d x+\frac{1}{r-1} \int_{Q_{0, T}}\left|u^{m}\right|^{r-1}\left|D_{t}\right| d x d t \leq \int_{Q_{0, T}}\left[\varkappa_{3}\left|u^{m}\right|^{r}+C_{22}\left(\varkappa_{3}\right)\left|D_{t}\right|^{r}\right] d x d t, \quad \varkappa_{3}>0 .
\end{gathered}
$$

By generalized Young's inequality with $q^{\prime}(x), q(x)>1$, it follows that

$$
\left|G u^{m} D\right| \leq g^{0}\left|u^{m}\right|^{q(x)-1}|D| \leq \varkappa_{4}\left|u^{m}\right|^{q(x)}+C_{23}\left(\varkappa_{4}\right)|D|^{q(x)}, \quad \varkappa_{4}>0 .
$$

Taking into account the Cauchy-Bunyakowski-Schwarz inequality, the Young inequality with $r^{\prime}, r>1$, and (16), we get

$$
\begin{gathered}
\left|\int_{Q_{0, T}} \phi\left(E u^{m}\right) D d x d t\right| \leq \phi^{*}|| E u^{m} ; L^{2}\left(Q_{0, T}\right)\|\cdot\| D ; L^{2}\left(Q_{0, T}\right) \| \\
\leq \phi^{*} E^{*}\left\|u^{m} ; L^{2}\left(Q_{0, T}\right)\right\| \cdot\left\|D ; L^{2}\left(Q_{0, T}\right)\right\| \leq \varkappa_{5} \int_{Q_{0, T}}\left|u^{m}\right|^{2} d x d t+C_{24}\left(\varkappa_{5}\right) \int_{Q_{0, T}}|D|^{2} d x d t, \quad \varkappa_{5}>0 .
\end{gathered}
$$

Here $C_{22}, \ldots, C_{24}>0$ are independent of $m$.

By (U) and (F), it follows that $\int_{\Omega}\left|u_{0}\right|^{r} d x+\int_{Q_{0, T}}\left[|f|^{2}+|D|^{2}+|D|^{q(x)}+\left|D_{t}\right|^{r}+\left|D_{t}\right|^{2}+|\nabla D|^{2}\right] d x d t \leq C_{25}$. Therefore, (77) yields that

$$
\begin{aligned}
& \mathscr{L}_{\varepsilon}\left(u^{m}, z^{m}\right)-\int_{Q_{0, T}} f z^{m} d x d t \geq \frac{1}{r} \int_{\Omega}\left|u^{m}(T)\right|^{r} d x+\int_{Q_{0, T}}\left[\left(\varepsilon-\frac{\varepsilon}{2}\right)\left|u_{t}^{m}\right|^{2}+\left(\alpha_{0}-a \varkappa_{2}\right)\left|\nabla u^{m}\right|^{2}\right. \\
& \left.+\left(\alpha_{0}-\varkappa_{1}-\varkappa_{5}\right)\left|u^{m}\right|^{2}+\left(\alpha_{0}-\varkappa_{3}\right)\left|u^{m}\right|^{r}+\left(g_{0}-\varkappa_{4}\right)\left|u^{m}\right|^{q(x)}\right] d x d t-C_{26}\left(\varkappa_{1}, \ldots, \varkappa_{5}\right),
\end{aligned}
$$

where $C_{26}>0$ is independent of $m$. Choosing $\varkappa_{1}, \ldots, \varkappa_{5}>0$ small enough, we obtain the existence of the nonnegative constants $C_{27}$ and $C_{28}$ such that (see (77))

$$
(P(\varphi), \varphi)_{\mathbb{R}^{m}} \geq C_{27} \int_{Q_{0, T}}\left[\left|\nabla u^{m}\right|^{2}+\left|u^{m}\right|^{2}\right] d x d t-C_{28} \underset{|\varphi| \rightarrow+\infty}{\longrightarrow}+\infty .
$$

Thus, the Vishyk Lemma (see Proposition 8) implies that there exist a solution $\varphi_{1}^{m}, \ldots, \varphi_{m}^{m}$ of system (76), i.e. (75). 
Step 2. Multiplying (75) by $\varphi_{\mu}^{m}$ and summing in $\mu=\overline{1, m}$, we obtain

$$
\mathscr{L}_{\varepsilon}\left(u^{m}, z^{m}\right)=\int_{Q_{0, T}} f z^{m} d x d t
$$

Then $0=\mathscr{L}_{\mathcal{\varepsilon}}\left(u^{m}, z^{m}\right)-\int_{Q_{0, T}} f z^{m} d x d t$ and, using (78), we get

$$
\varepsilon \int_{Q_{0, T}}\left|u_{t}^{m}\right|^{2} d x d t+\int_{\Omega}\left|u^{m}(T)\right|^{r} d x+\int_{Q_{0, T}}\left[\left|\nabla u^{m}\right|^{2}+\left|u^{m}\right|^{2}+\left|u^{m}\right|^{r}+\left|u^{m}\right|^{q(x)}\right] d x d t \leq C_{29} .
$$

By (79) and Proposition 3, we have

$$
\left\|u^{m} ; L^{q(x)}\left(Q_{0, T}\right)\right\| \leq C_{30} .
$$

Taking into account Lemma 2, (79), and (80), we obtain

$$
\begin{gathered}
\left\|G u^{m} ; L^{q^{\prime}(x)}\left(Q_{0, T}\right)\right\| \leq C_{31}, \quad\left\|\mathscr{R} u^{m} ; L^{r^{\prime}}\left(Q_{0, T}\right)\right\| \leq C_{32}, \\
\left\|\mathscr{R} u^{m}(T) ; L^{r^{\prime}}(\Omega)\right\| \leq C_{33} .
\end{gathered}
$$

Here $C_{29}, \ldots, C_{33}>0$ are independent of $m, \varepsilon$.

Estimates (79)-(82) yield that there exists a subsequence $\left\{u^{m_{j}}\right\}_{j \in \mathbb{N}} \subset\left\{u^{m}\right\}_{m \in \mathbb{N}}$ such that

$$
\begin{gathered}
u^{m_{j}} \underset{j \rightarrow \infty}{\longrightarrow} u \text { weakly in } H^{1}\left(Q_{0, T}\right) \cap L^{r}\left(Q_{0, T}\right) \cap L^{q(x)}\left(Q_{0, T}\right), \\
G u^{m_{j}} \underset{j \rightarrow \infty}{\longrightarrow} \widetilde{\chi}_{1} \text { weakly in } L^{q^{\prime}(x)}\left(Q_{0, T}\right), \\
\mathscr{R} u^{m_{j}} \underset{j \rightarrow \infty}{\longrightarrow} \widetilde{\chi}_{2} \text { weakly in } L^{r^{\prime}}\left(Q_{0, T}\right), \\
\mathscr{R} u^{m_{j}}(T) \underset{j \rightarrow \infty}{\longrightarrow} \widetilde{\chi}_{3} \text { weakly in } L^{r^{\prime}}(\Omega) .
\end{gathered}
$$

By (83), the Rellich-Kondrachov theorem (see Lemma 1.28 [27, p. 47]), and Lemma 1.18 [27, p. 39], it follows that there exists a subsequence (we call it $\left\{u^{m_{j}}\right\}_{j \in \mathbb{N}}$ again) such that

$$
u^{m_{j}} \underset{j \rightarrow \infty}{\longrightarrow} u \text { strongly in } L^{2}\left(Q_{0, T}\right) \text { and almost everywhere in } Q_{0, T} .
$$

By the Aubin theorem (see Proposition 7), we get

$$
u^{m_{j}} \underset{j \rightarrow \infty}{\longrightarrow} u \text { in } C\left([0, T] ; L^{2}(\Omega)\right) .
$$

Therefore, since $E$ is continuous and $\phi$ satisfies the Lipschitz condition, we obtain

$$
\phi\left(E u^{m_{j}}\right) \underset{j \rightarrow \infty}{\longrightarrow} \phi(E u) \text { strongly in } L^{2}\left(Q_{0, T}\right) .
$$

In addition,

$$
u^{m_{j}}(T) \underset{j \rightarrow \infty}{\longrightarrow} u(T) \text { strongly in } L^{2}(\Omega) \text { and almost everywhere in } \Omega \text {. }
$$


By (87), it follows that $G u^{m_{j}} \underset{j \rightarrow \infty}{\longrightarrow} G u$ almost everywhere in $Q_{0, T}$. Hence, Proposition 4 yields that $\tilde{\chi}_{1}=G u$. Continuing in the same way, we see that $\widetilde{\chi}_{2}=\mathscr{R} u$ and $\widetilde{\chi}_{3}=\mathscr{R} u(T)$.

Using (75) with $m=m_{j}$, (74), and the condition $\left.v^{\mu}\right|_{t=0}=0$, we obtain

$$
\int_{Q_{0, T}}\left[\varepsilon u_{t}^{m_{j}} v_{t}^{\mu}-\mathscr{R} u^{m_{j}} v_{t}^{\mu}+a\left(\nabla u^{m_{j}}, \nabla v^{\mu}\right)+G u^{m_{j}} v^{\mu}+\phi\left(E u^{m_{j}}\right) v^{\mu}\right] d x d t+\int_{\Omega} \mathscr{R} u^{m_{j}}(T) v^{\mu}(T) d x=\int_{Q_{0, T}} f v^{\mu} d x d t
$$

where $\mu=\overline{1, m_{j}}$. Letting $j \rightarrow \infty$, taking into account the properties of $\left\{v^{\mu}\right\}_{\mu \in \mathbb{N}}$ and (91), we get (66). Inequalities $r^{\prime}>2$, $q^{\prime}(x) \geq 2$ for a.e. $x \in \Omega,(16)$, and (81) imply that $\mathscr{R} u, G u, E u \in L^{2}\left(Q_{0, T}\right)$. Thus, $u$ is a weak solution to problem (62)-(63).

Step 3. Now we shall prove the additional estimates. By definition, put

$$
\mathscr{N} w:=G w+\phi(E w), \quad w \in L^{2}\left(Q_{0, T}\right)
$$

By inequalities $r^{\prime}>2, q^{\prime}(x) \geq 2$ for a.e. $x \in \Omega$, (16), and (81), it follows that

$$
\int_{Q_{0, T}}\left[\left|\mathscr{R} u^{m}\right|^{2}+\left|\mathscr{N} u^{m}\right|^{2}\right] d x d t \leq C_{34}
$$

where $C_{34}>0$ is independent of $m, \varepsilon$.

We recall that (74) holds, $\frac{3}{2}<r \leq 2$, and $u^{m} \in H^{2}\left(Q_{0, T}\right)$. Whence, using (61) with $p=2$ (note that $p \geq 2(r-1)$ ), we obtain

$$
\left\|\left(\left|u^{m}\right|^{r-2} u^{m}\right)_{t} ; L^{2}\left(Q_{0, T}\right)\right\| \leq C_{35}\left(\left\|u^{m} ; L^{2}\left(Q_{0, T}\right)\right\|^{r-1}+\left\|u_{t}^{m} ; L^{2}\left(Q_{0, T}\right)\right\|^{r-1}+\left\|u_{t t}^{m} ; L^{2}\left(Q_{0, T}\right)\right\|^{r-1}\right),
$$

where $C_{35}>0$ is independent of $m$. By (30) and (73, $)$, it follows that

$$
\left\|u_{t}^{m} ; L^{2}\left(Q_{0, T}\right)\right\|^{2} \leq C_{36} \int_{0}^{T}\left[\int_{\Omega}\left|u_{t}^{m}(x, T)\right|^{2} d x+\left\|u_{t t}^{m} ; L^{2}\left(Q_{0, T}\right)\right\|^{2}\right] d t=C_{37}\left\|u_{t t}^{m} ; L^{2}\left(Q_{0, T}\right)\right\|^{2}
$$

Thus, taking into account (79), the Young inequality with $\frac{1}{r-1}, \frac{1}{2-r}>1$, and (94), we get

$$
\left\|\left(\mathscr{R} u^{m}\right)_{t} ; L^{2}\left(Q_{0, T}\right)\right\| \leq \varkappa_{1}\left\|u_{t t}^{m} ; L^{2}\left(Q_{0, T}\right)\right\|+C_{38}\left(\varkappa_{1}\right), \quad \varkappa_{1}>0
$$

where $C_{38}\left(\varkappa_{1}\right)>0$ is independent of $m, \varepsilon$.

Step 4. Assume that the positive numbers $\left\{\widehat{\sigma_{m}}\right\}_{m \in \mathbb{N}}$ are determined from Remark 1. Hence, in particular, (28) hold. Using (733) and the condition $\left.v^{\mu}\right|_{t=0}=0$, we have

$$
\int_{Q_{0, T}} \varepsilon u_{t}^{m} v_{t}^{\mu} d x d t=\left.\int_{\Omega} \varepsilon u_{t}^{m} v^{\mu} d x\right|_{t=0} ^{t=T}-\int_{Q_{0, T}} \varepsilon u_{t t}^{m} v^{\mu} d x d t=-\int_{Q_{0, T}} \varepsilon u_{t t}^{m} v^{\mu} d x d t
$$


Then we multiply (75) by $\widehat{\sigma_{\mu}} \varphi_{\mu}^{m}$, use (28), and sum in $\mu=\overline{1, m}$. Taking into account (74) and (71), we obtain

$$
\int_{Q_{0, T}}\left[-\varepsilon u_{t t}^{m}\left(D_{t t}-u_{t t}^{m}\right)+\left(\mathscr{R} u^{m}\right)_{t}\left(D_{t t}-u_{t t}^{m}\right)+a\left(\nabla u^{m}, \nabla\left(-z_{t t}^{m}\right)\right)+\mathscr{N} u^{m}\left(D_{t t}-u_{t t}^{m}\right)\right] d x d t=\int_{Q_{0, T}} f\left(D_{t t}-u_{t t}^{m}\right) d x d t .
$$

The Young inequality and condition $(\Phi)$ imply that

$$
\begin{gathered}
\left|u_{t t}^{m} D_{t t}\right| \leq \frac{1}{2}\left|u_{t t}^{m}\right|^{2}+\frac{1}{2}\left|D_{t t}\right|^{2}, \quad\left|f D_{t t}\right| \leq \frac{1}{2}|f|^{2}+\frac{1}{2}\left|D_{t t}\right|^{2}, \quad\left|f u_{t t}^{m}\right| \leq \varkappa_{2}\left|u_{t t}^{m}\right|^{2}+\frac{1}{4 \varkappa_{2}}|f|^{2}, \quad \varkappa_{2}>0, \\
\left|\left(\mathscr{R} u^{m}\right)_{t}\left(D_{t t}-u_{t t}^{m}\right)\right| \leq \frac{\varkappa_{3}}{2}\left|u_{t t}^{m}\right|^{2}+C_{39}\left(\varkappa_{3}\right)\left(\left|\left(\mathscr{R} u^{m}\right)_{t}\right|^{2}+\left|D_{t t}\right|^{2}\right), \quad \varkappa_{3}>0, \\
\left|\mathscr{N} u^{m}\left(D_{t t}-u_{t t}^{m}\right)\right| \leq \varkappa_{4}\left|u_{t t}^{m}\right|^{2}+C_{40}\left(\varkappa_{4}\right)\left(\left|\mathscr{N} u^{m}\right|^{2}+\left|D_{t t}\right|^{2}\right), \quad \varkappa_{4}>0 .
\end{gathered}
$$

By (97) and (95) with $\varkappa_{1}>0$ small enough, it follows that

$$
\left|\int_{Q_{0, T}}\left(\mathscr{R} u^{m}\right)_{t}\left(D_{t t}-u_{t t}^{m}\right) d x d t\right| \leq \varkappa_{3} \int_{Q_{0, T}}\left|u_{t t}^{m}\right|^{2} d x d t+C_{41}\left(\varkappa_{3}\right)\left(1+\int_{Q_{0, T}}\left|D_{t t}\right|^{2} d x d t\right) .
$$

Since $\partial \Omega \in C^{4}$, Remark 1 yields that $\left\{v^{m}\right\}_{m \in \mathbb{N}} \subset H^{4}\left(Q_{0, T}\right)$. Integrating by parts and using $\left(72_{2,3}\right)$, we get

$$
\begin{gathered}
\int_{Q_{0, T}} a\left(\nabla u^{m},-\nabla z_{t t}^{m}\right) d x d t=-\int_{Q_{0, T}} a\left(\nabla D+\nabla z^{m}, \nabla z_{t t}^{m}\right) d x d t=I_{1}-\int_{Q_{0, T}} a\left(\nabla z^{m}, \nabla z_{t t}^{m}\right) d x d t \\
=I_{1}-\left.\int_{\Omega} a\left(\nabla z^{m}, \nabla z_{t}^{m}\right) d x\right|_{t=0} ^{t=T}+\int_{Q_{0, T}} a\left|\nabla z_{t}^{m}\right|^{2} d x d t=I_{1}+\int_{Q_{0, T}} a\left|\nabla u_{t}^{m}-\nabla D_{t}\right|^{2} d x d t \\
\geq I_{1}+\frac{a}{2} \int_{Q_{0, T}}\left|\nabla u_{t}^{m}\right|^{2} d x d t-C_{42} \int_{Q_{0, T}}\left|\nabla D_{t}\right|^{2} d x d t
\end{gathered}
$$

where

$$
I_{1}:=-\int_{Q_{0, T}} a\left(\nabla D, \nabla z_{t t}^{m}\right) d x d t
$$

Integrating by parts in $t$, using $\left(72_{3}\right)$, and using $\left(73_{2}\right)$, we obtain

$$
I_{1}=-\left.\int_{\Omega} a\left(\nabla D, \nabla z_{t}^{m}\right) d x\right|_{t=0} ^{t=T}+\int_{Q_{0, T}} a\left(\nabla D_{t}, \nabla z_{t}^{m}\right) d x d t=I_{2}+\int_{Q_{0, T}} a\left(\nabla D_{t}, \nabla z_{t}^{m}\right) d x d t
$$

where

$$
I_{2}:=\int_{\Omega} a\left(\nabla D(0), \nabla z_{t}^{m}(0)\right) d x
$$

Integrating by parts in $x$ and using condition (U), we get

$$
I_{2}=\int_{\partial \Omega} a(\nabla D(0), v) z_{t}^{m}(0) d S-\int_{\Omega} a(\Delta D(0)) z_{t}^{m}(0) d x=-\int_{\Omega} a z_{t}^{m}(0) \Delta D(0) d x .
$$


Moreover, $\left|a\left(\nabla D_{t}, \nabla z_{t}^{m}\right)\right|=\left|a\left(\nabla D_{t}, \nabla u_{t}^{m}-\nabla D_{t}\right)\right| \leq \varkappa_{5}\left|\nabla u_{t}^{m}\right|^{2}+C_{43}\left(\varkappa_{5}\right)\left|\nabla D_{t}\right|^{2}$. Whence,

$$
\left|I_{1}\right| \leq \int_{\Omega}\left[\varkappa_{6}\left|z_{t}^{m}(0)\right|^{2}+C_{44}\left(\varkappa_{6}\right)|\Delta D(0)|^{2}\right] d x+\int_{Q_{0, T}}\left[\varkappa_{5}\left|\nabla u_{t}^{m}\right|^{2}+C_{45}\left(\varkappa_{5}\right)\left|\nabla D_{t}\right|^{2}\right] d x d t, \quad \varkappa_{5}, \varkappa_{6}>0
$$

By the estimate of type (30), $\left(72_{3}\right)$, and (71), it follows that

$$
\int_{\Omega}\left|z_{t}^{m}(0)\right|^{2} d x \leq C_{46}\left(\int_{\Omega}\left|z_{t}^{m}(T)\right|^{2} d x+\int_{Q_{0, T}}\left|z_{t t}^{m}\right|^{2} d x d t\right)=C_{46} \int_{Q_{0, T}}\left|u_{t t}^{m}-D_{t t}\right|^{2} d x d t \leq C_{47} \int_{Q_{0, T}}\left[\left|u_{t t}^{m}\right|^{2}+\left|D_{t t}\right|^{2}\right] d x d t
$$

where $C_{47}>0$ is independent of $m$.

Finally, from (98) we get

$$
\begin{gathered}
\int_{Q_{0, T}} a\left(\nabla u^{m},-\nabla z_{t t}^{m}\right) d x d t \geq \int_{Q_{0, T}}\left[\left(\frac{a}{2}-\varkappa_{5}\right)\left|\nabla u_{t}^{m}\right|^{2}-C_{47} \varkappa_{6}\left|u_{t t}^{m}\right|^{2}\right] d x d t \\
-C_{48}\left(\varkappa_{5}, \varkappa_{6}\right)\left(\int_{\Omega}|\Delta D(0)|^{2} d x+\int_{Q_{0, T}}\left[\left|\nabla D_{t}\right|^{2}+\left|D_{t t}\right|^{2}\right] d x d t\right) .
\end{gathered}
$$

Since $\int_{\Omega}|\Delta D(0)|^{2} d x+\int_{Q_{0, T}}\left[|f|^{2}+\left|D_{t t}\right|^{2}+\left|\nabla D_{t}\right|^{2}\right] d x d t \leq C_{49}$, by (93), (95), and (96), it follows that

$$
\int_{Q_{0, T}}\left[\left(\varepsilon-\frac{\varepsilon}{2}-\varkappa_{2}-\varkappa_{3}-\varkappa_{4}-C_{47} \varkappa_{6}\right)\left|u_{t t}^{m}\right|^{2}+\left(\frac{a}{2}-\varkappa_{5}\right)\left|\nabla u_{t}^{m}\right|^{2}\right] d x d t \leq C_{50}\left(\varkappa_{2}, \ldots, \varkappa_{6}\right) .
$$

Hence, choosing $\varkappa_{2}, \ldots, \varkappa_{6}>0$ small enough, we have

$$
\int_{Q_{0, T}}\left[\left|u_{t t}^{m}\right|^{2}+\left|\nabla u_{t}^{m}\right|^{2}\right] d x d t \leq C_{51}
$$

Thus, (95) yields that

$$
\left\|\left(\mathscr{R} u^{m}\right)_{t} ; L^{2}\left(Q_{0, T}\right)\right\| \leq C_{52}
$$

Here $C_{50}, \ldots, C_{52}>0$ are independent of $m$.

Step 5. Assume that the positive numbers $\left\{\widehat{\lambda_{m}}\right\}_{m \in \mathbb{N}}$ are determined from Remark 1. Hence, in particular, (28) hold. Clearly (here and below $v$ denotes the outward unit vector field on $\partial \Omega$ ),

$$
\int_{Q_{0, T}} a\left(\nabla u^{m}, \nabla v^{\mu}\right) d x d t=\int_{\Sigma_{0, T}} a\left(\nabla u^{m}, v\right) v^{\mu} d S d t-\int_{Q_{0, T}} a \Delta u^{m} v^{\mu} d x d t=-\int_{Q_{0, T}} a \Delta u^{m} v^{\mu} d x d t
$$

Multiply (75) by $\widehat{\lambda_{\mu}} \varphi_{\mu}^{m}$, use (28) and (74), and sum in $\mu=\overline{1, m}$. Then we get

$$
\begin{gathered}
\int_{Q_{0, T}}\left[\varepsilon u_{t}^{m}\left(-\Delta z_{t}^{m}\right)+\left(\mathscr{R} u^{m}\right)_{t}\left(\Delta D-\Delta u^{m}\right)-a \Delta u^{m}\left(\Delta D-\Delta u^{m}\right)\right. \\
\left.\quad+\mathscr{N} u^{m}\left(\Delta D-\Delta u^{m}\right)\right] d x d t=\int_{Q_{0, T}} f\left(\Delta D-\Delta u^{m}\right) d x d t .
\end{gathered}
$$


Clearly (we recall that $\left\{z^{m}\right\}_{m \in \mathbb{N}} \subset H^{4}\left(Q_{0, T}\right)$ ),

$$
\begin{gathered}
-\int_{Q_{0, T}} \varepsilon u_{t}^{m} \Delta z_{t}^{m} d x d t=-\int_{\Sigma_{0, T}} \varepsilon u_{t}^{m}\left(\nabla z_{t}^{m}, v\right) d S d t+\int_{Q_{0, T}} \varepsilon\left(\nabla u_{t}^{m}, \nabla z_{t}^{m}\right) d x d t \\
\quad=\int_{Q_{0, T}} \varepsilon\left(\nabla u_{t}^{m}, \nabla u_{t}^{m}-\nabla D_{t}\right) d x d t \geq \frac{\varepsilon}{2} \int_{Q_{0, T}}\left[\left|\nabla u_{t}^{m}\right|^{2}-\left|\nabla D_{t}\right|^{2}\right] d x d t .
\end{gathered}
$$

The Young inequalities yields that

$$
\begin{gathered}
|f \Delta D| \leq \frac{1}{2}|f|^{2}+\frac{1}{2}|\Delta D|^{2}, \quad\left|a \Delta u^{m} \Delta D\right| \leq \varkappa_{1}\left|\Delta u^{m}\right|^{2}+\frac{a^{2}}{4 \varkappa_{1}}|\Delta D|^{2}, \quad \varkappa_{1}>0, \\
\left|f \Delta u^{m}\right| \leq \varkappa_{2}\left|\Delta u^{m}\right|^{2}+\frac{1}{4 \varkappa_{2}}|f|^{2}, \quad \varkappa_{2}>0, \\
\left|\left(\mathscr{R} u^{m}\right)_{t}\left(\Delta D-\Delta u^{m}\right)\right| \leq \varkappa_{3}\left|\Delta u^{m}\right|^{2}+C_{53}\left(\varkappa_{3}\right)\left(\left|\left(\mathscr{R} u^{m}\right)_{t}\right|^{2}+|\Delta D|^{2}\right), \quad \varkappa_{3}>0, \\
\left|\mathscr{N} u^{m}\left(\Delta D-\Delta u^{m}\right)\right| \leq \varkappa_{4}\left|\Delta u^{m}\right|^{2}+C_{54}\left(\varkappa_{4}\right)\left(\left|\mathscr{N} u^{m}\right|^{2}+|\Delta D|^{2}\right), \quad \varkappa_{4}>0 .
\end{gathered}
$$

Since $\int_{Q_{0, T}}\left[|f|^{2}+|\Delta D|^{2}+\left|\nabla D_{t}\right|^{2}\right] d x d t \leq C_{55}$, by (93), (95), and (101), it follows that

$$
\int_{Q_{0, T}}\left[\frac{\varepsilon}{2}\left|\nabla u_{t}^{m}\right|^{2}+\left(a-\varkappa_{1}-\varkappa_{2}-\varkappa_{3}-\varkappa_{4}\right)\left|\Delta u^{m}\right|^{2}\right] d x d t \leq C_{56}\left(\varkappa_{1}, \varkappa_{2}, \varkappa_{3}, \varkappa_{4}\right),
$$

where $C_{56}>0$ is independent of $m$. Therefore, choosing $\varkappa_{1}, \ldots, \varkappa_{4}>0$ small enough, we obtain

$$
\int_{Q_{0, T}}\left|\Delta u^{m}\right|^{2} d x d t \leq C_{57}
$$

where $C_{57}>0$ is independent of $m$.

By (100) and (87), it follows that

$$
\left(\mathscr{R} u^{m_{j}}\right)_{t} \underset{j \rightarrow \infty}{\longrightarrow}(\mathscr{R} u)_{t} \text { weakly in } L^{2}\left(Q_{0, T}\right)
$$

By (99) and (102), we get

$$
u^{m_{j}} \underset{j \rightarrow \infty}{\longrightarrow} u \text { weakly in } H^{2}\left(Q_{0, T}\right)
$$

and so $z^{m_{j}} \underset{j \rightarrow \infty}{\longrightarrow} z$ weakly in $H^{2}\left(Q_{0, T}\right)$. Since every function $z^{m}$ satisfies (72), the convergence above implies that $z \in U_{0}\left(Q_{0, T}\right)$. Thus, $u-D=z \in U_{0}\left(Q_{0, T}\right)$ and Theorem 6 is proved.

\section{Proof of main Theorem}

The Case $r=2$ is trivial. Assume that $r \in(1,2)$. The solution will be constructed via the elliptic regularization method.

Step 1. For every $\varepsilon>0$ let us denote by $u^{\varepsilon}$ a weak solution to problem (62)-(63) (see Theorem 6). Then (66) holds, $u^{\varepsilon} \in H^{2}\left(Q_{0, T}\right) \cap H^{1}\left(0, T ; H_{0}^{1}(\Omega)\right), \mathscr{R} u^{\varepsilon} \in H^{1}\left(0, T ; L^{2}(\Omega)\right)$, and $G u^{\varepsilon}, \phi\left(E u^{\varepsilon}\right) \in L^{2}\left(Q_{0, T}\right)$. By (79) and (80), we get

$$
\varepsilon \int_{Q_{0, T}}\left|u_{t}^{\varepsilon}\right|^{2} d x d t+\int_{Q_{0, T}}\left[\left|\nabla u^{\varepsilon}\right|^{2}+\left|u^{\varepsilon}\right|^{2}+\left|u^{\varepsilon}\right|^{r}+\left|u^{\varepsilon}\right|^{q(x)}\right] d x d t \leq C_{58},
$$




$$
\left\|u^{\varepsilon} ; L^{q(x)}\left(Q_{0, T}\right)\right\| \leq C_{59}
$$

Whence, similarly to (81), we obtain

$$
\left\|G u^{\varepsilon} ; L^{q^{\prime}(x)}\left(Q_{0, T}\right)\right\| \leq C_{60}, \quad\left\|\mathscr{R} u^{\varepsilon} ; L^{r^{\prime}}\left(Q_{0, T}\right)\right\| \leq C_{61} .
$$

In addition, by (16), $(\Phi)$, and (104), it follows that

$$
\left\|\phi\left(E u^{\varepsilon}\right) ; L^{2}\left(Q_{0, T}\right)\right\| \leq C_{62}
$$

Here $C_{58}, \ldots, C_{62}>0$ are independent of $\varepsilon$.

Estimates (104)-(107) implies that there exists a sequence $\left\{\varepsilon_{j}\right\}_{j \in \mathbb{N}} \subset \mathbb{R}_{+}$such that $\varepsilon_{j} \underset{j \rightarrow \infty}{\longrightarrow} 0$ and

$$
\begin{aligned}
& u^{\varepsilon_{j}} \underset{j \rightarrow \infty}{\longrightarrow} u \text { weakly in } L^{2}\left(0, T ; H_{0}^{1}(\Omega)\right) \cap L^{r}\left(Q_{0, T}\right) \cap L^{q(x)}\left(Q_{0, T}\right), \\
& \qquad u^{\varepsilon_{j}} \underset{j \rightarrow \infty}{\longrightarrow} \chi_{1} \text { weakly in } L^{q^{\prime}(x)}\left(Q_{0, T}\right), \\
& \mathscr{R} u^{\varepsilon_{j}} \underset{j \rightarrow \infty}{\longrightarrow} \chi_{2} \text { weakly in } L^{r^{\prime}}\left(Q_{0, T}\right), \\
& \phi\left(E u^{\varepsilon_{j}}\right) \underset{j \rightarrow \infty}{\longrightarrow} \chi_{3} \text { weakly in } L^{2}\left(Q_{0, T}\right), \\
& \sqrt{\varepsilon_{j}} u_{t}^{\varepsilon_{j}} \underset{j \rightarrow \infty}{\longrightarrow} \chi_{4} \text { weakly in } L^{2}\left(Q_{0, T}\right) .
\end{aligned}
$$

Step 2. Notice that the constants in (99), (100), and (102) depend on $\varepsilon$ and so we can not use these estimates here. We shall prove the additional estimates.

Since $u^{\varepsilon} \in H^{2}\left(Q_{0, T}\right)$ and $2 \geq 2\left(\frac{r}{2}+1-1\right)$ holds, Case 2.ii of Theorem 5 with $p=2$ and $\frac{r}{2}+1$ instead of $r$ yields that

$$
\left|u^{\varepsilon}\right|^{\frac{r}{2}-1} u^{\varepsilon} \in H^{1}\left(0, T ; L^{2}(\Omega)\right), \quad\left(\left|u^{\varepsilon}\right|^{\frac{r}{2}-1} u^{\varepsilon}\right)_{t}=\frac{r}{2}\left|u^{\varepsilon}\right|^{\frac{r}{2}-1} u_{t}^{\varepsilon} .
$$

Since $u^{\varepsilon} \in H^{2}\left(Q_{0, T}\right)$ and $\frac{3}{2}<r \leq 2$ holds, similarly to (74), we have

$$
\mathscr{R} u^{\varepsilon} \in H^{1}\left(0, T ; L^{2}(\Omega)\right), \quad\left(\mathscr{R} u^{\varepsilon}\right)_{t}=\left|u^{\varepsilon}\right|^{r-2} u_{t}^{\varepsilon} .
$$

Moreover, $u^{\varepsilon} \in H^{1}\left(Q_{0, T}\right), 2 \geq r$, and so similarly to (70), we obtain

$$
\left|u^{\varepsilon}\right|^{r} \in W^{1, \frac{2}{r}}\left(0, T ; L^{\frac{2}{r}}(\Omega)\right), \quad\left(\left|u^{\varepsilon}\right|^{r}\right)_{t}=r\left|u^{\varepsilon}\right|^{r-2} u^{\varepsilon} u_{t}^{\varepsilon} .
$$

Continuing in the same way (we recall that $u^{\varepsilon}, u_{t}^{\varepsilon}, u_{x_{1}}^{\varepsilon}, \ldots, u_{x_{n}}^{\varepsilon} \in H^{1}\left(Q_{0, T}\right)$ ), using Case 1.ii of Theorem 2 with $p(x) \equiv 2$ and $r(x) \equiv 2$, we see that

$$
\begin{gathered}
\left|u^{\varepsilon}\right|^{2} \in W^{1,1}\left(0, T ; L^{1}(\Omega)\right), \quad\left(\left|u^{\varepsilon}\right|^{2}\right)_{t}=2 u^{\varepsilon} u_{t}^{\varepsilon}, \\
\left|u_{t}^{\varepsilon}\right|^{2} \in W^{1,1}\left(0, T ; L^{1}(\Omega)\right), \quad\left(\left|u_{t}^{\varepsilon}\right|^{2}\right)_{t}=2 u_{t}^{\varepsilon} u_{t t}^{\varepsilon}, \\
\left|\nabla u^{\varepsilon}\right|^{2} \in W^{1,1}\left(0, T ; L^{1}(\Omega)\right), \quad\left(\left|\nabla u^{\varepsilon}\right|^{2}\right)_{t}=2\left(\nabla u^{\varepsilon}, \nabla u_{t}^{\varepsilon}\right) .
\end{gathered}
$$


Finally, since $u^{\varepsilon} \in H^{1}\left(Q_{0, T}\right)$ and $q^{0} \leq 2$ holds, Case 1 .ii of Theorem 2 with $r(x)=q(x)$ and $p(x) \equiv 2$ yields that

$$
\left|u^{\varepsilon}\right|^{q(x)},\left(\left|u^{\varepsilon}\right|^{q(x)}\right)_{t} \in L^{\frac{2}{q(x)}}\left(Q_{0, T}\right), \quad\left(\left|u^{\varepsilon}\right|^{q(x)}\right)_{t}=q(x)\left|u^{\varepsilon}\right|^{q(x)-2} u^{\varepsilon} u_{t}^{\varepsilon} .
$$

Integrating by parts (see (114) ) and using (633), we obtain

$$
\begin{gathered}
\int_{Q_{0, T}}\left[\varepsilon u_{t}^{\varepsilon} v_{t}-\mathscr{R} u^{\varepsilon} v_{t}\right] d x d t=\left.\int_{\Omega}\left[\varepsilon u_{t}^{\varepsilon} v-\mathscr{R} u^{\varepsilon} v\right] d x\right|_{t=0} ^{t=T}-\int_{Q_{0, T}}\left[\varepsilon u_{t t^{\varepsilon}} v-\left(\mathscr{R} u^{\varepsilon}\right)_{t} v\right] d x d t \\
=-\int_{\Omega} \mathscr{R} u^{\varepsilon}(T) v(T) d x+\int_{Q_{0, T}}\left[-\varepsilon u_{t t^{\varepsilon}}^{\varepsilon}+\left(\mathscr{R} u^{\varepsilon}\right)_{t} v\right] d x d t, \quad v \in U_{0}\left(Q_{0, T}\right) .
\end{gathered}
$$

Then from (66) it follows that

$$
\int_{Q_{0, T}}\left[-\varepsilon u_{t t}^{\varepsilon} v+\left(\mathscr{R} u^{\varepsilon}\right)_{t} v+a\left(\nabla u^{\varepsilon}, \nabla v\right)+G u^{\varepsilon} v+\phi\left(E u^{\varepsilon}\right) v\right] d x d t=\int_{Q_{0, T}} f v d x d t, \quad v \in U_{0}\left(Q_{0, T}\right) .
$$

Assume that $w \in H_{0}^{1}(\Omega), \varphi \in C^{1}([0, T])$, and $\varphi(0)=0$. Substituting $v(x, t)=w(x) \varphi(t)$ in (120), we get

$$
\int_{0}^{T}\left(\int_{\Omega_{t}}\left[-\varepsilon u_{t t}^{\varepsilon} w+\left(\mathscr{R} u^{\varepsilon}\right)_{t} w+a\left(\nabla u^{\varepsilon}, \nabla w\right)+G u^{\varepsilon} w+\phi\left(E u^{\varepsilon}\right) w-f w\right] d x\right) \varphi(t) d t=0 .
$$

Take a function $\psi \in C_{0}^{\infty}((0, T))$. Substituting $\varphi=\psi$ in $(121)$, we have the following equality in $H^{-1}(\Omega):=\left[H_{0}^{1}(\Omega)\right]^{*}$ :

$$
-\varepsilon u_{t t}^{\varepsilon}(t)+\left(\mathscr{R} u^{\varepsilon}(t)\right)_{t}+A u^{\varepsilon}(t)+G u^{\varepsilon}(t)+\phi\left(E u^{\varepsilon}(t)\right)=f(t), \quad t \in(0, T),
$$

where the operator $A: H_{0}^{1}(\Omega) \rightarrow H^{-1}(\Omega)$ is defined by the rule

$$
\langle A v, w\rangle_{H_{0}^{1}(\Omega)}=\int_{\Omega} a(\nabla v(x), \nabla w(x)) d x, \quad v, w \in H_{0}^{1}(\Omega) .
$$

Since $u_{t}^{\varepsilon} \in L^{2}\left(0, T ; H_{0}^{1}(\Omega)\right)$, by (122) and (114), it follows that

$$
\int_{Q_{0, T}}\left[-\varepsilon u_{t t}^{\varepsilon} u_{t}^{\varepsilon}+\left|u^{\varepsilon}\right|^{r-2}\left|u_{t}^{\varepsilon}\right|^{2}+a\left(\nabla u^{\varepsilon}, \nabla u_{t}^{\varepsilon}\right)+G u^{\varepsilon} u_{t}^{\varepsilon}+\phi\left(E u^{\varepsilon}\right) u_{t}^{\varepsilon}\right] d x d t=\int_{Q_{0, T}} f u_{t}^{\varepsilon} d x d t .
$$

Integrating by parts (see (117) ) and using (633), we obtain

$$
\int_{Q_{0, T}}-\varepsilon u_{t t}^{\varepsilon} u_{t}^{\varepsilon} d x d t=-\left.\frac{\varepsilon}{2} \int_{\Omega}\left|u_{t}^{\varepsilon}\right|^{2} d x\right|_{t=0} ^{t=T}=-0+\frac{\varepsilon}{2} \int_{\Omega}\left|u_{t}^{\varepsilon}(0)\right|^{2} d x \geq 0 .
$$

From the Young inequality (note that $\frac{1}{2}+\frac{1}{r^{\prime}}+\frac{r^{\prime}-2}{2 r^{\prime}}=1$ ), it follows that

$$
\begin{gathered}
\left|f u_{t}^{\varepsilon}\right|=\left|u^{\varepsilon}\right|^{\frac{r}{2}-1}\left|u_{t}^{\varepsilon}\right||f|\left|u^{\varepsilon}\right|^{1-\frac{r}{2}} \leq \varkappa_{1}\left|u^{\varepsilon}\right|^{r-2}\left|u_{t}^{\varepsilon}\right|^{2}+C_{63}\left(\varkappa_{1}\right)\left(|f|^{r^{\prime}}+\left|u^{\varepsilon}\right|^{\left(1-\frac{r}{2}\right) \frac{2 r^{\prime}}{r^{\prime}-2}}\right) \\
=\varkappa_{1}\left|u^{\varepsilon}\right|^{r-2}\left|u_{t}^{\varepsilon}\right|^{2}+C_{63}\left(\varkappa_{1}\right)\left(|f|^{r^{\prime}}+\left|u^{\varepsilon}\right|^{r}\right), \quad \varkappa_{1}>0,
\end{gathered}
$$


Assume that the constant $\gamma \in\left(\frac{4}{3}, 2\right]$ is defined by (11). Then the Young inequality with $\frac{4-r}{2}, \frac{4-r}{2-r}>1$ implies that

$$
\left|u_{t}^{\varepsilon}\right|^{\gamma}=\left|u^{\varepsilon}\right|^{\frac{2(r-2)}{4-r}}\left|u_{t}^{\varepsilon}\right|^{\frac{4}{4-r}}\left|u^{\varepsilon}\right|^{\frac{2(2-r)}{4-r}} \leq \varkappa_{2}\left|u^{\varepsilon}\right|^{r-2}\left|u_{t}^{\varepsilon}\right|^{2}+C_{64}\left(\varkappa_{2}\right)\left|u^{\varepsilon}\right|^{2}, \quad \varkappa_{2}>0 .
$$

Whence, since $\phi$ is bounded, the Young inequality and (104) yield that

$$
\left|\int_{Q_{0, T}} \phi\left(E u^{\varepsilon}\right) u_{t}^{\varepsilon} d x d t\right| \leq C_{65} \int_{Q_{0, T}}\left|u_{t}^{\varepsilon}\right| d x d t \leq \int_{Q_{0, T}}\left|u_{t}^{\varepsilon}\right|^{\gamma} d x d t+C_{66} \leq \varkappa_{Q_{0, T}}\left|u^{\varepsilon}\right|^{r-2}\left|u_{t}^{\varepsilon}\right|^{2} d x d t+C_{67}\left(\varkappa_{2}\right) .
$$

Taking into account the transformations above, by (124), (118), and (119), we obtain

$$
\int_{Q_{0, T}}\left[\left(1-\varkappa_{1}-\varkappa_{2}\right)\left|u^{\varepsilon}\right|^{r-2}\left|u_{t}^{\varepsilon}\right|^{2}+\frac{a}{2}\left(\left|\nabla u^{\varepsilon}\right|^{2}\right)_{t}+\frac{g(x, t)}{q(x)}\left(\left|u^{\varepsilon}\right|^{q(x)}\right)_{t}\right] d x d t \leq C_{68}\left(\varkappa_{1}, \varkappa_{2}\right) .
$$

Choosing $\varkappa_{1}, \varkappa_{2}>0$ small enough and integrating by parts, we get

$$
\int_{\Omega_{T}}\left[\frac{a}{2}\left|\nabla u^{\varepsilon}\right|^{2}+\frac{g_{0}}{q^{0}}\left|u^{\varepsilon}\right|^{q(x)}\right] d x+\frac{1}{2} \int_{Q_{0, T}}\left|u^{\varepsilon}\right|^{r-2}\left|u_{t}^{\varepsilon}\right|^{2} d x d t \leq C_{69}\left(1+\int_{\Omega}\left[\left|\nabla u_{0}\right|^{2}+\left|u_{0}\right|^{q(x)}\right] d x+\int_{Q_{0, T}} \frac{\left|g_{t}(x, t)\right|}{q(x)}\left|u^{\varepsilon}\right|^{q(x)} d x d t\right) .
$$

Thus,

$$
\int_{Q_{0, T}}\left|u^{\varepsilon}\right|^{r-2}\left|u_{t}^{\varepsilon}\right|^{2} d x d t \leq C_{70}
$$

and so (113) yields that

$$
\int_{Q_{0, T}}\left|\left(\left|u^{\varepsilon}\right|^{\frac{r}{2}-1} u^{\varepsilon}\right)_{t}\right|^{2} d x d t=\frac{|r|^{2}}{4} \int_{Q_{0, T}}\left|u^{\varepsilon}\right|^{r-2}\left|u_{t}^{\varepsilon}\right|^{2} d x d t \leq C_{71} .
$$

Moreover, from (104) it follows that

$$
\left.\left.\int_{Q_{0, T}}|| u^{\varepsilon}\right|^{\frac{r}{2}-1} u^{\varepsilon}\right|^{2} d x d t=\int_{Q_{0, T}}\left|u^{\varepsilon}\right|^{r} d x d t \leq C_{72} .
$$

In addition, (125), (127), and (104) imply that

$$
\int_{Q_{0, T}}\left|u_{t}^{\varepsilon}\right|^{\gamma} d x d t \leq C_{73}
$$

Here $C_{63}, \ldots, C_{73}>0$ are independent of $\varepsilon$.

Estimates (104) and (127)-(130) yield that

$$
\begin{gathered}
\left|u^{\varepsilon_{j}}\right|^{\frac{r}{2}-1} u^{\varepsilon_{j}} \underset{j \rightarrow \infty}{\longrightarrow} \chi_{5} \text { weakly in } H^{1}\left(0, T ; L^{2}(\Omega)\right), \\
u^{\varepsilon_{j}} \underset{j \rightarrow \infty}{\longrightarrow} u \text { weakly in } W^{1, \gamma}\left(Q_{0, T}\right) .
\end{gathered}
$$

Hence, using the Rellich-Kondrachov theorem (see Lemma 1.28 [27, p. 47]) and Lemma 1.18 [27, p. 39], we obtain

$$
u^{\varepsilon_{j}} \underset{j \rightarrow \infty}{\longrightarrow} u \text { strongly in } L^{\gamma}\left(Q_{0, T}\right) \text { and almost everywhere in } Q_{0, T}
$$


Therefore, (see Proposition 4, (109), (110), and (131) ) $\chi_{1}=G u, \chi_{2}=\mathscr{R} u$, and $\chi_{5}=|u|^{\frac{r}{2}-1} u$. By (132) and the Aubin theorem (see Proposition 7), it follows that

$$
u^{\varepsilon_{j}} \underset{j \rightarrow \infty}{\longrightarrow} u \text { in } C\left([0, T] ; L^{\gamma}(\Omega)\right)
$$

It is easy to prove that the operator $E$ (see (3) ) is continuous from $L^{\gamma}\left(Q_{0, T}\right)$ into $L^{\gamma}\left(Q_{0, T}\right)$. Then, $(\Phi)$ and (134) imply that $\phi\left(E u^{\varepsilon_{j}}\right) \underset{j \rightarrow \infty}{\longrightarrow} \phi(E u)$ strongly in $L^{\gamma}\left(Q_{0, T}\right)$. Thus, $\chi_{3}=\phi(E u)$.

Step 3. We substitute $\varepsilon=\varepsilon_{j}$ in (66) and tend $j \rightarrow \infty$ if $v \in H_{0}^{1}\left(Q_{0, T}\right)$. Taking into account (108)-(112), we obtain (15). By (132) and condition (63), it follows that the function $u$ satisfies (2). Thus, $u$ is a weak solution to problem (1)-(2). By (131) and (132), we get $|u|^{\frac{r}{2}-1} u \in H^{1}\left(0, T ; L^{2}(\Omega)\right)$ and $u \in W^{1, \gamma}\left(Q_{0, T}\right)$. This completes the proof of Theorem 1 .

\section{Competing interests}

The authors declare that they have no competing interests.

\section{Authors' contributions}

All authors have contributed to all parts of the article. All authors read and approved the final manuscript.

\section{References}

[1] R.A. Adams. Sobolev spaces. Academic Press, New York, San Francisco, London, 1975.

[2] S. Antontsev, S. Shmarev. Evolution PDEs with nonstandard growth conditions. Existence, uniqueness, localization, blow-up. Atlantis Studies in Diff. Eq., Vol. 4, Paris: Atlantis Press, 2015.

[3] J.-P. Aubin. Un theoreme de compacite, Comptes rendus hebdomadaires des seances de l'academie des sciences. 256, No 24 (1963) 5042-5044.

[4] T.A. Averina, K.A. Rybakov. New methods of analysis of the Poisson delta-impulse in problem of radiotechnics, J. of Radioelectronics. 1 (2013) 1-20.

[5] F. Bernis. Existence results for doubly nonlinear higher order parabolic equations on unbounded domains, Math. Ann. 279 (1988) 373-394.

[6] M. Bokalo, V. Dmytriv. Boundary value problems for integro-differential equations in asicotropic spaces, Visnyk (Herald) of Lviv Univ. Series Mech.-Math. 59 (2001) 84-101.

[7] T.M. Bokalo, O.M. Buhrii. Doubly nonlinear parabolic equations with variable exponents of nonlinearity, Ukr. Math. J. 63, No 5 (2011) 709-728 (Translated from Ukr. Mat. Z. 63, No 5 (2011) 612-628).

[8] H. Brezis. Functional Analysis, Sobolev Spaces and Partial Differential Equations. Springer, New York, Dordrecht, Heidelberg, London, 2011.

[9] M. Briani, R. Natalini, G. Russo. Implicit-explicit numerical schemes for jump-diffusion processes, Calcolo. 44, No 1 (2007) 33-57.

[10] O.M. Buhrii. Parabolic variational inequalities without initial conditions, Ph. D. thesis (Lviv, Ukraine, 2001).

[11] O.M. Buhrii. Finiteness of time vanishing of the solution of a nonlinear parabolic variational inequality with variable exponent of nonlinearity, Mat. Studii. 24, No 2 (2005) 167-172.

[12] O.M. Buhrii On integration by parts formulaes for special type of exponential functions, Mat. Studii. 45, No 2 (2016) 118-131.

[13] O. Buhrii, M. Buhrii. On existence in generalized Sobolev spaces the solution of the initial-boundary value problem for nonlinear integro-differential equations arising from theory of European option, Visnyk (Herald) of Lviv Univ. Series Mech.-Math., 2016 (accepted). 
[14] J. Byström. Sharp Constants for Some Inequalities Connected to The p-Laplace Operator, Jour. of Ineq. in Pure and Appl. Math. 6, No 2 (2005) Article 56.

[15] P. Carr, L. Wu. Time-changed Levy processes and option pricing, J. of Financial Economics. 71 (2004) 113-141.

[16] C. la Chioma. Integro-differential problems arising in pricing derivatives in jump-diffusion markets. Ph.D. Thesis. (Roma, 20032004).

[17] M. Chipot, A. Rougirel. On some class of problems with nonlocal source and boundary flux, Adv. Differential Equations. 6, No 9 (2001) 1025-1048.

[18] M. Chipot, N.-H. Chang. On some model diffusion problems with a nonlocal lower order term, Chin. Ann. Math. 24, No 2 (2003) 147-166.

[19] M. Chipot, N.-H. Chang. Nonlinear nonlocal evolution problems, Rev. R. Acad. Cien. Serie A. Mat. 97, No 3 (2003) $423-445$.

[20] S. Cifani, E.R. Jakobsen, K.H. Karlsen. The discontinuous Galerkin method for fractional degenerate convection-diffusion equations, BIT. 51, No 4 (2011) 809-844.

[21] S.S. Clift. Linear and non-linear monotone methods for valuing financial options under two-factor, jump-diffusion models, Ph.D. thesis in Computer Science. (Waterloo, Ontario, Canada, 2007).

[22] L. Diening, P. Harjulehto, P. Hasto, M. Ruzicka. Lebesgue and Sobolev spaces with variable exponents. Springer, Heidelberg, 2011.

[23] Yu.A. Dubinskii. Quasylinear elliptic and parabolic equations of any order, Uspekhi Mat. Nauk. Vol. 23, No 1 (139) (1968) $45-90$.

[24] N. Dunford, J.T. Schwartz. Linear operators. Part 1: General theory. Izd. Inostran Lit., Moscow, 1962. (translated from: Interscience Publ., New York, London, 1958).

[25] L.C. Evans. Partial differential equations. Graduate Studies in Mathematics. Amer. Math. Soc., Providence, RI, 1998.

[26] X.-L. Fan, D. Zhao. On the spaces $L^{p(x)}(\Omega)$ and $W^{m, p(x)}(\Omega)$, J. Math. Anal. Appl. 263 (2001) 424-446.

[27] H. Gajewski, K. Groger, K. Zacharias. Nonlinear operator equations and operator differential equations. Mir, Moscow, 1978. (translated from: Akademie-Verlag, Berlin, 1974).

[28] D. Kinderlehrer, G. Stampacchia. Introduction to variational inequalities and its applications. Mir, Moscow, 1983. (translated from: Academic Press, New York, London, Toronto, Sydney, San Francisco, 1980).

[29] A.N. Kolmogorov, S.V. Fomin. Elements of theory of functions and functional analysis. Nauka, Moscow, 1972.

[30] O. Kováčik, J. Rákosník. On spaces $L^{p(x)}$ and $W^{1, p(x)}$, Czechoslovak Math. J. 41 (116) (1991) 592-618.

[31] S.G. Kou. A jump-diffusion model for option pricing, Management Science. 48 (2002) 1086-1101.

[32] O.A. Ladyzhenskaya, N.N. Ural'tseva. Linear and quasilinear elliptic equations, 2th edition. Nauka, Moscow, 1973.

[33] J.-L. Lions. Some methods of solving of nonlinear boundary value problems. Mir, Moscow, 1972. (translated from: Dunod, Gauthier-Villars, Paris, 1969).

[34] R.C. Merton. Option pricing when underlying stock returns are discontinuous, J. of Financial Economics. 3 (1976) 125-144.

[35] V.P. Mikhailov. Partial differential equations. Nauka, Moscow, 1976.

[36] W. Orlicz. Uber Konjugierte Exponentenfolgen. Studia Mathematica (Lviv) 3 (1931) 200-211.

[37] O.T. Panat. Problems for hyperbolic equations and hyperbolic-parabolic systems in generalized Sobolev spaces, Ph.D. thesis (Lviv, Ukraine, 2010).

[38] J.P. Pinasco. Blow-up for parabolic and hyperbolic problems with variable exponents, Nonlinear Analysis. 71 (2009) 1094-1099.

[39] A. Rougirel. Blow-up rate for parabolic problems with nonlocal source and boundary flux, Electronic J. of Diff. Eq. 2003, No 98 (2003) 1-18.

[40] Ph. Souplet. Uniform blow-up profiles and boundary behavior for diffusion equations with nonlocal nonlinear source, J. Diff. Equations. 153 (1999) 374-406.

[41] T.P. Timsina. Sensitivities in option pricing models, Ph.D. thesis in Mathematics. (Blacksberg, Virginia, USA, 2007).

[42] E.C. Titchmarch. Eigenfunction expansions associated with second-order differential equations. Part II. (Clarendon Press, Oxford, 1958). 